\title{
Performance Analysis and Optimal Allocation of Layered Defense M/M/N Queueing Systems
}

\author{
Longyue Li, ${ }^{1}$ Fuxian Liu, ${ }^{1}$ Guangzheng Long, ${ }^{1}$ Huizhen Zhao, ${ }^{1}$ and Yingying Mei ${ }^{2}$ \\ ${ }^{1}$ Air and Missile Defense College, Air Force Engineering University, Xian 710051, China \\ ${ }^{2}$ Chongqing Nankai Secondary School, Chongqing 400030, China \\ Correspondence should be addressed to Longyue Li; lilong-yue@hotmail.com
}

Received 9 May 2016; Accepted 3 October 2016

Academic Editor: Anna Pandolfi

Copyright (C) 2016 Longyue Li et al. This is an open access article distributed under the Creative Commons Attribution License, which permits unrestricted use, distribution, and reproduction in any medium, provided the original work is properly cited.

\begin{abstract}
One important mission of strategic defense is to develop an integrated layered Ballistic Missile Defense System (BMDS). Motivated by the queueing theory, we presented a work for the representation, modeling, performance simulation, and channels optimal allocation of the layered BMDS M/M/N queueing systems. Firstly, in order to simulate the process of defense and to study the Defense Effectiveness (DE), we modeled and simulated the M/M/N queueing system of layered BMDS. Specifically, we proposed the $\mathrm{M} / \mathrm{M} / \mathrm{N} / \mathrm{N}$ and $\mathrm{M} / \mathrm{M} / \mathrm{N} / \mathrm{C}$ queueing model for short defense depth and long defense depth, respectively; single target channel and multiple target channels were distinguished in each model. Secondly, we considered the problem of assigning limited target channels to incoming targets, we illustrated how to allocate channels for achieving the best DE, and we also proposed a novel and robust search algorithm for obtaining the minimum channel requirements across a set of neighborhoods. Simultaneously, we presented examples of optimal allocation problems under different constraints. Thirdly, several simulation examples verified the effectiveness of the proposed queueing models. This work may help to understand the rules of queueing process and to provide optimal configuration suggestions for defense decision-making.
\end{abstract}

\section{Introduction}

These years, ballistic missile (BM) technology has spread to more and more countries. Nations all over the world are developing missiles capable of reaching enemies. One important mission of strategic defense is to develop an integrated layered BMDS to defend homeland, deployed forces, allies, and friends from ballistic missile attacks [1]. The BMDS is based on a multilayer defense concept and therefore contains more than one defense weapon; it will include different types of defense weapons located on land or ships used to destroy ballistic missiles [2]. Layered BMDS has two advantages: (1) interception mainly can be divided into 3 phases: boost phase, midphase, and reentry phase. Since the reentry phase is too short and it is the last chance for a shot, BMDS should not rely on a single defense weapon but on defense weapons placed at different locations forming a layered BMDS; the layered BMDS allows for more shot opportunities that will certainly increase the probability of a successful interception
[3]. (2) For given affordable BMs penetration probability (or expected kill probability), cooperation between different missile defense weapons may reduce the expected resources consumption and provide an efficient way of using interceptors. The common methods used in the research on the process simulation and performance evaluation of missile defense are the mathematical programming method $[4,5]$, the probability calculation method [6], the system simulation method [7], the Markov method [8, 9], and so forth.

Queueing theory is a mathematical theory of stochastic service system which was first proposed by Erlang [10]. Queueing systems have a wide range of applications, such as resource allocation [11], system optimization [12], and communication planning [13]. Similarly, in order to make full use of defense capabilities, queuing theory also has a lot of applications in defense weapons operation research; it can solve problems of weapons configuration or efficiency analysis [14-16]. Following are the two questions that need our attention: (1) there are many factors that affect DE, such 
as the number of layers, the number of defense weapons, and Single Shot Kill Probability (SSKP); these are also factors that affect the requirement of defense weapons; how are defense weapons, number of layers, BMs, SSKP, and DE interrelated and how can we understand this relationship for achieving the best allocation plan? (2) If we have deployed different types of defense weapons, then how do we deal with them?

Using $\mathrm{M} / \mathrm{M} / \mathrm{N}$ queueing system to simulate the missile defense process is feasible; the reasons are as follows: (1) the Poisson process has the simplest mathematical expressions, though BMs arrival is not fully consistent with the Poisson process; it represents the most difficult scenario (worstcase scenario) for the BMDS to deal with. As long as the BMDS can deal with Poisson arrivals, it has certain adaptability to other types of arrivals distribution. (2) BMs usually have fixed and highly predictable trajectories, though some of them may have limited maneuvering potential; we think this has no influence on our research. (3) The incoming directions, firing tactics, technical characteristics, and time intervals of BM arrivals have some Poisson features; these can be viewed as customers waiting to be served by servers. (4) The targets capacity (number of target channels, servers) and shooting times for each target (service times) are limited. When BMs that arrived find that all channels are occupied (not idle) or there was little time for a shot, they will penetrate the BMDS directly. In summary, the $\mathrm{M} / \mathrm{M} / \mathrm{N}$ queueing system can be used to analyze the DE of BMDS, summarize the rules of defense, and provide suggestions for system configuration for defense decision-making. The remainder of the paper is structured as follows. Section 2 proposes the framework for $M / M / N$ queueing model. Section 3 discusses $M / M / N$ queueing models. Section 4 is dedicated to optimal allocation of target channels. Section 5 provides numerical examples. Section 6 includes concluding remarks and future work.

\section{M/M/N Queueing Framework}

We consider an $\mathrm{M} / \mathrm{M} / \mathrm{N}$ queueing system with BMs arrival rate $\lambda$ and shooting rate $\mu$ of defense weapons. $M$ stands for "memoryless" or "Markovian" and means that the process being represented by $\mathrm{M}$ comes from an exponential distribution [17].

(1) Suppose that BMs arrive randomly and independently of each other to a defense weapon and that the average rate at which they arrive is given by the parameter $\lambda[18]$; that is,

$$
P_{n}(t)=\frac{(\lambda t)^{n}}{n !} e^{-\lambda t}, \quad t>0, n=0,1,2, \ldots
$$

This is known as a Poisson arrival process; $P_{n}(t)$ is the probability that $n$ BMs arrive within time $t$. Suppose that the time intervals between arrivals are randomly taken from the exponential distribution with parameter $\lambda$; their probability density function and distribution function are

$$
\begin{aligned}
& f_{T}(t)= \begin{cases}\lambda e^{-\lambda t}, & t \geq 0 \\
0, & t<0,\end{cases} \\
& F_{T}(t)= \begin{cases}1-\lambda e^{-\lambda t}, & t \geq 0 \\
0, & t<0 .\end{cases}
\end{aligned}
$$

The exponential distribution is memoryless, which indicates that the BMs arrivals are random.

(2) Suppose that the BMs are shot in the order of their arrivals; the shooting time for a $\mathrm{BM}$ is also exponentially distributed at rate $\mu$; then, its probability density function and distribution function are

$$
\begin{aligned}
& f_{s}(t)= \begin{cases}\mu e^{-\mu t}, & t \geq 0 \\
0, & t<0,\end{cases} \\
& F_{s}(t)= \begin{cases}1-\mu e^{-\mu t}, & t \geq 0 \\
0, & t<0,\end{cases}
\end{aligned}
$$

where $\mu=1 / t_{\text {smean }}$, where $t_{\text {smean }}$ is the mean shooting time. The shooting time depends on the reaction time of the defense weapon and the time of interceptor flying from the launch point to the calculated encounter point, which is related to the technical capabilities of defense weapons. Introducing $\rho=\lambda / \mu, \rho<1$ means that the queue is stable if the mean shooting time is less than the mean arrival time intervals; it can be understood as the firing density (shooting intensity) [19].

(3) Suppose that the waiting times of BMs are also exponentially distributed at rate $v$; then their probability density function and distribution function are

$$
\begin{aligned}
& f_{w}(t)= \begin{cases}v e^{-v t}, & t \geq 0 \\
0, & t<0,\end{cases} \\
& F_{w}(t)= \begin{cases}1-v e^{-v t}, & t \geq 0 \\
0, & t<0,\end{cases}
\end{aligned}
$$

where $\mu=1 / t_{w \text { mean }}$, where $t_{w \text { mean }}$ is the mean waiting time.

(4) Additionally, if there is an idle target channel when a $\mathrm{BM}$ arrives at the system, then the defense weapon will shoot it immediately. In this paper, we divided the queueing system into two types: (1) loss system (when BMs that arrived find that all target channels were occupied (not idle), they will penetrate the BMDS directly (leave the system without service)) and (2) mixed system (when BMs that arrived find that all target channels were occupied, BMs will not be penetrated but will wait for a limited time (depending on the time of BMs flying in the killing zone of BMDS) until a target channel becomes available). We use the term "defense depth" to distinguish between the loss system and the mixed system. "Short defense depth" is defined as the case when 


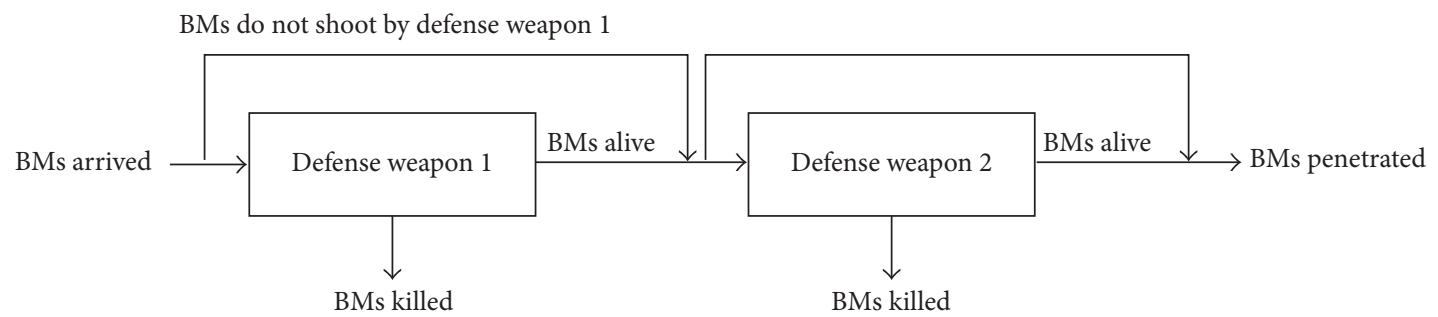

(a) Long defense depth

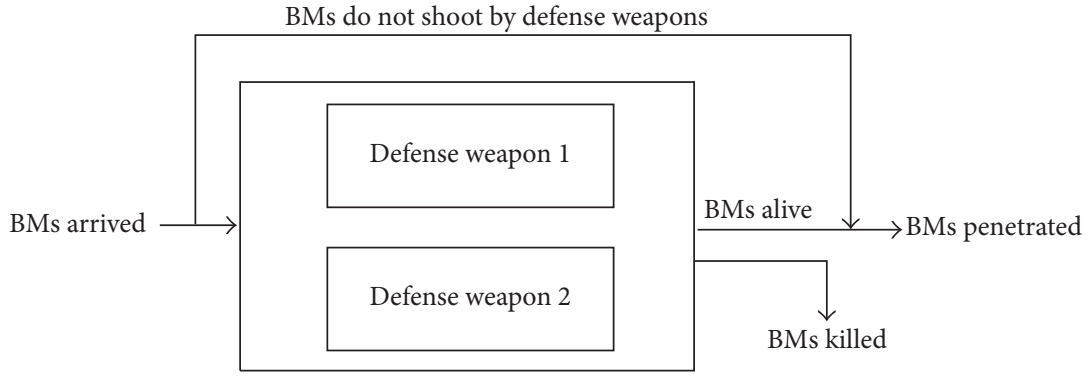

(b) Short defense depth

FIGURE 1: Long defense depth and short defense depth.

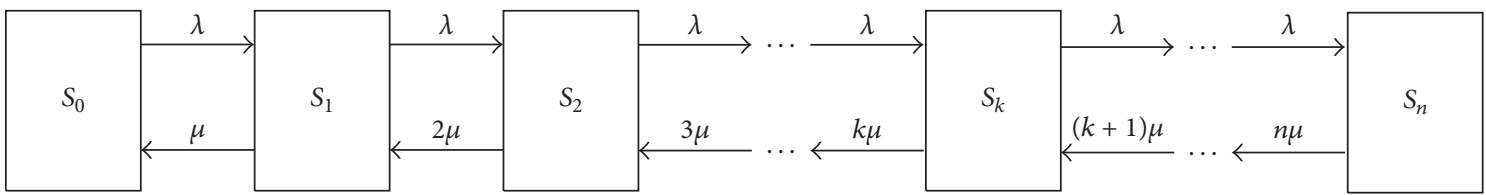

FIGURE 2: State transition diagram of M/M/N/N system.

waiting times of BMs are shorter than shooting times of defense weapons (loss system), and the "long defense depth" is defined as the case when waiting times of BMs are longer than shooting times of defense weapons (mixed system), as shown in Figure 1.

\section{M/M/N Queueing Models}

\subsection{Identical Weapons, Short Defense Depth, and $M / M / N / N$ System}

3.1.1. Multiple Target Channels and $M / M / N / N$ System. BMDS with short defense depth can be seen as a random loss system that does not wait. Let $n$ be the number of target channels of BMDS; that is, the number of BMs shot by defense weapons cannot exceed $n$ at the same time; $P_{f}$ is the detection probability of BMDS radars. Possible states of the system are as follows:

$S_{0}$ : all target channels are idle; there is no $\mathrm{BM}$ in the system.
$S_{1}: 1$ target channel is busy; there is $1 \mathrm{BM}$ in the system.

$S_{k}: k$ target channels are busy; there are $k$ BMs in the system.

$S_{n}$ : all target channels are busy; there are $n$ BMs in the system.

Figure 2 is the state transition diagram of $M / M / N / N$ system. In Figure 2, the arrow $\rightarrow$ indicates incoming BMs, and the arrival rate is $\lambda . S_{k}$ means $k$ target channels are busy, and the state is transferred to $S_{k+1}$ when a new BM arrives. Arrow $\leftarrow$ indicates shootings of defense weapons, when the shooting process for a $\mathrm{BM}$ is done, and then the state is transferred from $S_{1}$ to $S_{0}$ with rate $\mu$, transferred from $S_{2}$ to $S_{1}$ with rate $2 \mu, \ldots$, and transferred from $S_{k}$ to $S_{k-1}$ with rate $k \mu$. Balance equations are as follows: 
$S_{0}$ : all target channels are idle; there is no $\mathrm{BM}$ in the system:

$$
\lambda S_{0}=\mu S_{1} \Longrightarrow S_{1}=\left(\frac{\lambda}{\mu}\right) S_{0}=\rho S_{0}
$$

$S_{1}: 1$ target channel is busy; there is $1 \mathrm{BM}$ in the system:

$$
\lambda S_{1}=2 \mu S_{2} \Longrightarrow S_{2}=\left(\frac{\rho^{2}}{2 !}\right) S_{0} .
$$

$S_{2}: 2$ target channels are busy; there are $2 \mathrm{BMs}$ in the system:

$$
\lambda S_{2}=2 \mu S_{3} \Longrightarrow S_{3}=\left(\frac{\rho^{3}}{3 !}\right) S_{0}
$$

$S_{k-1}: k-1$ target channels are busy; there are $k-1 \mathrm{BMs}$ in the system:

$$
\lambda S_{k-1}=k \mu S_{k} \Longrightarrow S_{k}=\left(\frac{\rho^{k}}{k !}\right) S_{0} .
$$

$S_{n}: n$ target channels are busy; there are $n$ BMs in the system:

$$
\lambda S_{n-1}=n \mu S_{n} \Longrightarrow S_{n}=\left(\frac{\rho^{n}}{n !}\right) S_{0} .
$$

Due to the fact that $P_{f}$ is the probability of the BM found by the radar, we have

$$
\rho_{f}=\rho \cdot P_{f}=P_{f} \cdot\left(\frac{\lambda}{\mu}\right)=P_{f} \cdot \lambda \cdot t_{\text {smean }} .
$$

$\rho_{f}$ is the mean number of BMs found to arrive during the mean shooting time. Because $S_{0}+S_{1}+S_{2}+\cdots+S_{n}=1$, then the expression for $S_{0}$ can be written in the form

$$
\begin{aligned}
S_{0} & =\left(1+\rho_{f}+\frac{\rho_{f}^{2}}{2 !}+\cdots+\frac{\rho_{f}^{k}}{k !}+\cdots+\frac{\rho_{f}^{n}}{n !}\right)^{-1} \\
& =\left(\sum_{k=0}^{n} \frac{\rho_{f}^{k}}{k !}\right)^{-1} .
\end{aligned}
$$

The optimal operation of the queueing system can be analyzed through several performance parameters, some of which follow.

(1) BM loss probabilities (penetration probabilities):

$$
P_{\text {lost }}=S_{n}=\left(\frac{\rho_{f}^{n}}{n !}\right) S_{0}=\left(\frac{\rho_{f}^{n}}{n !}\right)\left(\sum_{k=0}^{n} \frac{\rho_{f}^{k}}{k !}\right)^{-1} .
$$

(2) Relative probabilities of BMs that will be shot:

$$
P_{\text {shoot }}=1-P_{\text {lost }}=1-\left(\frac{\rho_{f}^{n}}{n !}\right)\left(\sum_{k=0}^{n} \frac{\rho_{f}^{k}}{k !}\right)^{-1} .
$$

(3) Absolute probabilities of BMs that will be shot:

$$
P_{\text {shoot }}^{\prime}=\lambda P_{\text {shoot }}=\lambda\left(1-\left(\frac{\rho_{f}^{n}}{n !}\right)\left(\sum_{k=0}^{n} \frac{\rho_{f}^{k}}{k !}\right)^{-1}\right) \text {. }
$$

(4) Mean number of occupied target channels:

$$
\begin{aligned}
\bar{Z} & =0 \cdot S_{0}+1 \cdot S_{1}+\cdots+n \cdot S_{n}=\sum_{k=0}^{n} k\left(\frac{\rho_{f}{ }^{k}}{k !}\right) S_{0} \\
& =\rho_{f} S_{0}\left(\sum_{k=0}^{n} \frac{\rho_{f}{ }^{k}}{k !}-\frac{\rho_{f}{ }^{n}}{n !}\right) \\
& =\rho_{f}\left(1-\frac{\rho_{f}{ }^{n}}{n !}\left(\sum_{k=0}^{n} \frac{\rho_{f}^{k}}{k !}\right)^{-1}\right) .
\end{aligned}
$$

The mean occupancy rate of target channels is $\eta=\bar{Z} / n$.

3.1.2. Single Target Channel and $M / M / 1 / 1$ System. The $\mathrm{M} / \mathrm{M} / 1 / 1$ queueing system can be viewed as a special case of Section 3.1.1 for $n=1$; then, the expression for $S_{0}$ can be rewritten in the form

$$
S_{0}=\left.\left(\sum_{k=0}^{n} \frac{\rho_{f}^{k}}{k !}\right)^{-1}\right|_{n=1}=\frac{1}{1+\rho_{f}} .
$$

Performance parameters are as follows:

(1) BM loss probabilities:

$$
P_{\text {lost }}=S_{n}=\left(\frac{\rho_{f}^{n}}{n !}\right) S_{0}=\frac{\rho_{f}}{1+\rho_{f}} .
$$

(2) Relative probabilities of BMs that will be shot:

$$
P_{\text {shoot }}=1-P_{\text {lost }}=1-\frac{\rho_{f}}{1+\rho_{f}}=\frac{1}{1+\rho_{f}} .
$$

(3) Absolute probabilities of BMs that will be shot:

$$
P_{\text {shoot }}^{\prime}=\lambda P_{\text {shoot }}=\frac{\lambda}{1+\rho_{f}} .
$$

The concept of DE is the product of probabilities of BM that will be shot times SSKP; that is,

$$
E=P_{\text {shoot }} \cdot P_{k}=\left(1-P_{\text {lost }}\right) \cdot P_{k} \text {. }
$$

3.2. Identical Weapons, Long Defense Depth, and $M / M / N / C$ System. From Section 1, we know that BMDS with long defense depth can be regarded as a stochastic service system with limited waiting time, that is, the mixed queueing system. For each incoming BM, the defense weapons use idle target channels to shoot. When BMs that arrived find that all target channels were occupied, BMs will not be penetrated but will wait for a limited time until a target channel becomes 
available. Possible states of the system are as follows:

$S_{0}$ : all target channels are idle; there is no $\mathrm{BM}$ in the system.

$S_{1}: 1$ target channel is busy; there is $1 \mathrm{BM}$ in the system.

$S_{k}: k$ target channels are busy; there are $k$ BMs in the system.

$\vdots$

$S_{n}$ : all target channels are busy; there are $n$ BMs in the system.
$S_{n+1}$ : all target channels are busy; there is $1 \mathrm{BM}$ waiting.

$S_{n+s}:$ all target channels are busy; there are $s$ BMs waiting.

Figure 3 is the state transition diagram of $M / M / N / C$ system. In Figure 3, the arrow $\rightarrow$ indicates incoming BMs, and the arrival rate is $\lambda . S_{k}$ means $k$ target channels are busy, and the state is transferred from lower state to higher state when new BMs arrive. Arrow $\leftarrow$ indicates shootings of defense weapons, when the shooting process for a BM is done, and then the state is transferred from higher state to lower state with rate $k \mu$ or $n \mu+s \nu$. Balance equations are

$$
\begin{array}{l|l}
S_{0} & 0=-\lambda S_{0}+\mu S_{1} \\
S_{1} & 0=\lambda S_{0}-(\lambda+\mu) S_{1}+2 \mu S_{2} \\
\vdots & \vdots \\
S_{k} & 0=\lambda S_{k-1}-(\lambda+k \mu) S_{k}+(k+1) \mu S_{2} \\
\vdots & \vdots \\
S_{n} & \left\{\begin{array}{l}
0=\lambda S_{n-1}-(\lambda+n \mu) S_{n}+(n \mu+\nu) S_{n+1} \\
S_{n+1} \\
\vdots \\
0=\lambda S_{n}-(\lambda+n \mu+\nu) S_{n+1}+(n \mu+2 \nu) S_{n+2} \\
S_{n+s} \\
\vdots \\
\vdots
\end{array}\right.
\end{array}
$$

Then, we have the constraint that $\sum_{i=0}^{\infty} S_{i}=1$ for (21). Let $\rho=\lambda / \mu$ be the mean number of BMs that arrived during the mean shooting time; let $\vartheta=\nu / \mu$ be the mean number of penetrated (leaving without being shot) BMs during the mean shooting time. Then, (21) can be written in the form

$$
\begin{array}{c|l}
S_{0} & 0=-\rho S_{0}+S_{1} \\
S_{1} & 0=\rho S_{0}-(\rho+1) S_{1}+2 S_{2} \\
\vdots & \vdots \\
S_{k} & 0=\rho S_{k-1}-(\rho+k) S_{k}+(k+1) S_{2} \\
\vdots & \vdots \\
S_{n} & 0=\rho S_{n-1}-(\rho+n) S_{n}+(n+\vartheta) S_{n+1} \\
S_{n+1} & 0=\rho S_{n}-(\rho+n+\vartheta) S_{n+1}+(n+2 \vartheta) S_{n+2} \\
\vdots & \vdots \\
S_{n+s} & 0=\rho S_{n+s-1}-(\rho+n+\vartheta) S_{n+s}+(n+(s+1) \vartheta) S_{n+s+1} \\
\vdots & \vdots
\end{array}
$$

When $k \leq n$, we have

$$
\begin{aligned}
S_{1} & =\left(\frac{\lambda}{\mu}\right) S_{0}=\rho S_{0}=\frac{\rho^{1}}{1 !} S_{0}, \\
S_{2} & =\frac{1}{2}\left((\rho+1) S_{1}-\rho S_{0}\right)=\frac{1}{2}\left(\rho S_{1}+S_{1}-\rho S_{0}\right) \\
& =\frac{\rho}{2} S_{1}=\frac{\rho^{2}}{2 !} S_{0} \\
S_{n} & =\frac{\rho}{n} S_{k-1}=\frac{\rho^{n}}{n !} S_{0} .
\end{aligned}
$$

When $k>n$, we have

$$
\begin{aligned}
S_{n+1} & =\frac{1}{n+\vartheta}\left((\rho+n) S_{n}-\rho S_{n-1}\right) \\
= & \frac{1}{n+\vartheta}\left(\rho S_{n}+n S_{n}-\rho S_{n-1}\right)=\frac{\rho}{n+\vartheta} S_{n}
\end{aligned}
$$




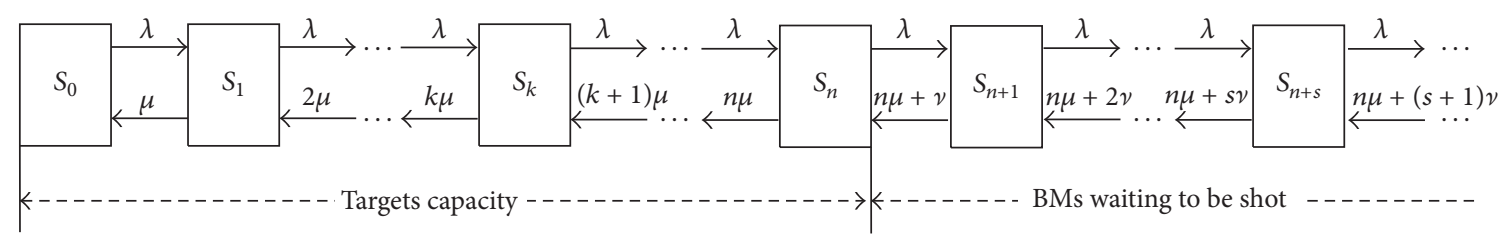

FIGURE 3: State transition diagram of M/M/N/C system.

$$
\begin{aligned}
\quad & \frac{\rho}{n+\vartheta} \frac{\rho^{n}}{n !} S_{0}=\frac{\rho^{n}}{n !} \frac{\rho}{n+\vartheta} S_{0}, \\
S_{n+2} & =\frac{1}{n+2 \vartheta}\left((\rho+(\rho+\vartheta)) S_{n+1}-\rho S_{n}\right) \\
& =\frac{1}{n+2 \vartheta}\left(\rho S_{n+1}+(\rho+\vartheta) S_{n+1}-\rho S_{n}\right) \\
& =\frac{1}{n+2 \vartheta}\left(\rho S_{n+1}+\rho S_{n}-\rho S_{n}\right) \\
& =\frac{\rho^{n}}{n !} \frac{\rho^{2}}{(n+\vartheta)(n+2 \vartheta)} S_{0} \\
S_{n+s} & =\frac{\rho}{n+s \vartheta} S_{n+s-1}=\frac{\rho^{n}}{n !} \frac{\rho^{s}}{\prod_{m=1}^{s}(n+m \vartheta)} S_{0} .
\end{aligned}
$$

The constraint for (24) is

$$
\sum_{m=0}^{n} S_{m}+\sum_{s=1}^{\infty} S_{m+s}=1
$$

With direct substitution of (23) and (24) into (25), it follows that

$$
\begin{gathered}
1=S_{0}\left(\sum_{m=0}^{n} \frac{\rho^{m}}{m !}+\frac{\rho^{n}}{n !} \sum_{s=1}^{\infty}\left(\rho^{s}\left(\prod_{m=1}^{s}(n+m \vartheta)\right)^{-1}\right)\right), \\
S_{0}=\left(\sum_{m=0}^{n} \frac{\rho^{m}}{m !}+\frac{\rho^{n}}{n !} \sum_{s=1}^{\infty}\left(\rho^{s}\left(\prod_{m=1}^{s}(n+m \vartheta)\right)^{-1}\right)\right)^{-1} .
\end{gathered}
$$

Then, with substitution of (26) into (24), we have steady-state probability

$$
\begin{aligned}
& S_{k}=\frac{\rho^{k}}{k !}\left(\sum_{m=0}^{n} \frac{\rho^{m}}{m !}+\frac{\rho^{n}}{n !} \sum_{s=1}^{\infty}\left(\rho^{s}\left(\prod_{m=1}^{s}(n+m \vartheta)\right)^{-1}\right)\right)^{-1}, \\
& S_{n+s} \\
& =\left(\frac{\left(\rho^{n} / n !\right)\left(\rho^{s}\left(\prod_{m=1}^{s}(n+m \vartheta)\right)^{-1}\right)}{\sum_{m=0}^{n}\left(\rho^{m} / m !\right)+\left(\rho^{n} / n !\right) \sum_{s=1}^{\infty}\left(\rho^{s}\left(\prod_{m=1}^{s}(n+m \vartheta)\right)^{-1}\right)}\right),
\end{aligned}
$$

Performance parameters are as follows:

(1) BM loss probabilities ( $s$ BMs waiting to be shot):

$$
\begin{aligned}
& P_{\text {lost }}=\frac{\nu}{\lambda} \sum_{s=1}^{\infty} s S_{n+s}=\frac{\nu / \mu}{\lambda / \mu} \sum_{s=1}^{\infty} s S_{n+s}=\frac{\vartheta}{\rho} \sum_{s=1}^{\infty} s S_{n+s}=\frac{\vartheta}{\rho} \\
& \cdot \sum_{s=1}^{\infty} \frac{s\left(\rho^{n} / n !\right)\left(\rho^{s}\left(\prod_{m=1}^{s}(n+m \vartheta)\right)^{-1}\right)}{\sum_{m=0}^{n}\left(\rho^{m} / m !\right)+\left(\rho^{n} / n !\right) \sum_{s=1}^{\infty}\left(\rho^{s}\left(\prod_{m=1}^{s}(n+m \vartheta)\right)^{-1}\right)} .
\end{aligned}
$$

(2) Relative probabilities of BMs that will be shot:

$$
P_{\text {shoot }}=1-P_{\text {lost }}
$$

(3) Absolute probabilities of BMs that will be shot:

$$
P_{\text {shoot }}^{\prime}=\lambda P_{\text {shoot }}
$$

(4) Mean number of occupied target channels:

$$
\begin{aligned}
\bar{Z} & =\sum_{k=1}^{n} k S_{k}+\sum_{s=1}^{\infty} n S_{n+s}=\sum_{k=1}^{n} k S_{k}+n \sum_{s=1}^{\infty} S_{n+s} \\
\text { or } \bar{Z} & =\frac{\lambda P_{\text {shoot }}}{\mu}=\rho P_{\text {shoot }} .
\end{aligned}
$$

The mean occupancy rate of target channels is

$$
\eta=\frac{\rho P_{\text {shoot }}}{n} .
$$

$\mathrm{DE}$ is the product of probabilities of BM that will be shot times SSKP; that is,

$$
E=P_{\text {shoot }} \cdot P_{k}=\left(1-P_{\text {lost }}\right) \cdot P_{k}
$$

Similarly, the $\mathrm{M} / \mathrm{M} / \mathrm{1} / \mathrm{C}$ queueing system can be viewed as a special case for $n=1$.

3.3. Different Defense Weapons. Defense weapons in Sections 3.1 and 3.2 are identical, and the BMDS may deploy different types of defense weapons. For different types of defense weapons, the waiting time of BMs, the detection probability of radars, and SSKP may be different. In order to model the queueing system, we choose one type of defense weapon as the reference and then substitute the reference defense (type $r$ ) for defense weapons of type $i$. The "replacement process" is 
named equivalent replacement method [20]; basic equations are

$$
\begin{aligned}
& \lambda_{(r)} \cdot P_{\text {shoot }(r)} \cdot P_{k(r)} \cdot P_{f(r)}=\lambda_{(i)} \cdot P_{\text {shoot }(i)} \cdot P_{k(i)} \cdot P_{f(i)}, \\
& n_{(r)} \cdot\left(1+t_{w \text { mean }(r)}\right) \cdot P_{k(r)} \\
& =n_{(i)} \cdot\left(1+t_{w \operatorname{mean}(i)}\right) \cdot P_{k(i)}, \\
& n_{(r)}=\frac{n_{(i)} \cdot\left(1+t_{w \operatorname{mean}(i)}\right) \cdot P_{k(i)}}{\left(1+t_{w \operatorname{mean}(r)}\right) \cdot P_{k(r)}} .
\end{aligned}
$$

Subscripts $(\cdot)_{(r)}$ and $(\cdot)_{(i)}$ in (34) are used to distinguish defense weapons of type $r$ from defense weapons of type $i$. From the above equations, we can substitute $D_{(r)}$ for $D_{(i)}$; the intensity of BMs killed by defense weapons of type $i$ and the intensity of BMs arrivals for defense weapons of type $i$ are

$$
\begin{aligned}
& \rho_{(i)}=\frac{D_{(i)} \cdot P_{f(i)} \cdot P_{k(i)}}{t_{w \operatorname{time}(i)}}, \\
& \lambda_{(i)}=\lambda \rho_{(i)} \cdot\left(\sum_{i=1}^{R} \rho_{(i)}\right)^{-1},
\end{aligned}
$$

respectively, where $R$ denotes the total number of types.

\section{Optimal Allocation of Target Channels}

\subsection{Identical Defense Weapons and $M / M / N / N$ System}

4.1.1. Defense Effectiveness. Let $M$ be the number of layers; the number of target channels deployed along the $i$ th layer is denoted by $n_{i}$; the probability of BMs that will be shot by the $M$-layer defense with short defense depth is

$$
P_{\text {shoot }}^{M}=1-\left(\frac{\rho^{N}}{N !}\right)\left(\sum_{k=0}^{N} \frac{\rho^{k}}{k !}\right)^{-1},
$$

where $N$ is the total number of target channels, $N=\sum_{i=1}^{M} n_{i}$. The probabilities of BMs that will be shot by the $M$ layer defense with short defense depth are as follows:

1st layer:

$$
\begin{aligned}
\rho_{1} & =\rho_{f}^{(1)}=\lambda \cdot t_{\text {wean }} \cdot P_{f}, \\
P_{\text {shoot }}^{(1)} & =1-\left(\frac{\rho_{1}^{n_{1}}}{n_{1} !}\right)\left(\sum_{k=0}^{n_{1}} \frac{\rho_{1}^{k}}{k !}\right)^{-1}, \\
E_{1} & =P_{\text {shoot }}^{(1)} \cdot P_{k} .
\end{aligned}
$$

2nd layer:

$$
\begin{aligned}
\rho_{2} & =\left(1-E_{1}\right) \cdot \lambda \cdot t_{\text {wean }} \cdot P_{f}=\left(1-E_{1}\right) \cdot \rho_{1}, \\
P_{\text {shoot }}^{(2)} & =1-\left(\frac{\rho_{2}{ }^{n_{2}}}{n_{2} !}\right)\left(\sum_{k=0}^{n_{2}} \frac{\rho_{2}{ }^{k}}{k !}\right)^{-1}, \\
E_{2} & =P_{\text {shoot }}^{(2)} \cdot P_{k} .
\end{aligned}
$$

Mth layer:

$$
\begin{aligned}
\rho_{M}= & \left(1-E_{M-1}\right)\left(1-E_{M-2}\right) \cdots\left(1-E_{1}\right) \lambda \cdot t_{w \text { mean }} \\
& \cdot P_{f}=\left(\prod_{i=1}^{M-1}\left(1-E_{i}\right)\right) \cdot \rho_{1} \\
= & \left(1-E_{M-1}\right) \cdot \rho_{M-1}, \\
P_{\text {shoot }}^{(M)}= & 1-\left(\frac{\rho_{M}^{n_{M}}}{n_{M} !}\right)\left(\sum_{k=0}^{n_{M}} \frac{\rho_{M}^{k}}{k !}\right)^{-1}, \\
E_{M}= & P_{\text {shoot }}^{(M)} \cdot P_{k} .
\end{aligned}
$$

DE of the whole $M$-layer BMDS is

$$
\begin{aligned}
E= & E_{1}+\left(1-E_{1}\right) E_{2}+\left(1-E_{1}\right)\left(1-E_{2}\right) E_{3} \cdots \\
& +\prod_{i=1}^{M-1}\left(1-E_{i}\right) E_{M} \\
= & E_{1}+\sum_{k=2}^{M}\left(E_{k} \cdot \prod_{i=1}^{M-1}\left(1-E_{i}\right)\right) .
\end{aligned}
$$

Then, we define the optimization problem as finding numbers of layers and target channels deployed along the $i$ th layer so as to maximize DE, subject to a given set of constraints:

$\max E$

$$
\begin{aligned}
= & E_{1}+\left(1-E_{1}\right) E_{2}+\left(1-E_{1}\right)\left(1-E_{2}\right) E_{3} \cdots \\
& +\prod_{i=1}^{M-1}\left(1-E_{i}\right) E_{M}
\end{aligned}
$$

s.t. $\rho_{i}$

$$
\begin{aligned}
& =\left(1-E_{i-1}\right)\left(1-E_{i-2}\right) \cdots\left(1-E_{1}\right) \lambda \cdot t_{\text {wmean }} \\
& \quad \cdot P_{f}=\left(\prod_{x=1}^{i-1}\left(1-E_{i}\right)\right) \cdot \rho_{1} \\
& =\left(1-E_{i-1}\right) \cdot \rho_{i-1}, \\
& P_{\text {shoot }}^{(i)}=1-\left(\frac{\rho_{i}^{n_{i}}}{n_{i} !}\right)\left(\sum_{k=0}^{n_{i}} \frac{\rho_{i}^{k}}{k !}\right)^{-1}, \\
& n=\sum_{i=1}^{M} n_{i}, \\
& E_{i}=P_{\text {shoot }}^{(i)} \cdot P_{k} .
\end{aligned}
$$

For the nonlinear optimization problem (41), when problem size is small, we can use algebra, dynamic programming, or enumeration method to solve it. When the size of the problem is very large, an approximate solution can be obtained by using some advanced algorithms, for example, genetic, neural network, and heuristic algorithms. In order to get some potential and useful allocation rules, we analyze a scenario. 
TABLE 1: DE of two-layer defense.

\begin{tabular}{|c|c|c|c|c|}
\hline 1st-layer channel number & 1st-layer DE & 2nd-layer channel number & 2nd-layer DE & Total DE \\
\hline 9 & $56.00 \%$ & 1 & $14.56 \%$ & $62.41 \%$ \\
\hline 8 & $56.00 \%$ & 2 & $25.20 \%$ & $67.09 \%$ \\
\hline 7 & $54.32 \%$ & 3 & $32.48 \%$ & $69.16 \%$ \\
\hline 6 & $52.08 \%$ & 4 & $41.44 \%$ & $70.94 \%$ \\
\hline 5 & $47.04 \%$ & 5 & $44.24 \%$ & $70.47 \%$ \\
\hline 4 & $35.84 \%$ & 6 & $44.80 \%$ & $64.58 \%$ \\
\hline 3 & $34.16 \%$ & 7 & $53.76 \%$ & $69.56 \%$ \\
\hline 2 & $27.44 \%$ & 8 & $54.88 \%$ & $67.26 \%$ \\
\hline 1 & $11.20 \%$ & 9 & $56.00 \%$ & $60.93 \%$ \\
\hline
\end{tabular}

Scenario 1. Suppose that the number of target channels is 10 , SSKP is 0.7 , the probability that BMs will be detected by radars is $0.8, \lambda=5 \mathrm{BMs} / \mathrm{min}$, and $t_{\text {smean }}=0.75 \mathrm{~min}$.

Tables 1, 2, and 3 are the DE of two-layer, three-layer, and four-layer defense, respectively.

Let $\mathbf{n}_{(\mathbf{M})}=\left(n_{1}, n_{2}, \ldots, n_{M}\right)$ be the allocation plan of target channels, where $M$ is the number of layers and $n_{i}$ is the number of target channels deployed along the $i$ th layer. From the results of Tables 1,2 , and 3 , we noticed that plans $\mathbf{n}_{(2)}=(6,4), \mathbf{n}_{(3)}=(5,3,2)$, and $\mathbf{n}_{(4)}=(4,3,2,1)$ have the biggest DE. We also found that $\mathbf{n}_{(2)}=(6,4)<\mathbf{n}_{(3)}=$ $(5,3,2)<\mathbf{n}_{(4)}=(4,3,2,1)$, and then we have Theorem 1 .

Theorem 1. Let $\mathbf{E}_{\left(\mathbf{M}, \mathbf{n}_{(\mathrm{M})}\right)}$ be the DE of the M-layer BMDS; $\mathbf{n}_{(\mathbf{M})}=\left(n_{1}, n_{2}, \ldots, n_{M}\right)$ is the allocation plan, $n_{i}$ is the number of target channels deployed along the ith layer, and $N=\sum_{i=1}^{M} n_{i}$, $n_{i} \geq 1$. When $N$ is constant, then $\max \mathbf{E}_{\left(\mathbf{M}, \mathbf{n}_{(\mathrm{M})}\right)}$ is stochastically increasing as $M$ increases; that is,

$$
\max E_{\left(M-1, n_{(M)}\right)} \leq \max E_{\left(M, n_{(M)}\right)} \leq \max E_{\left(M+1, n_{(M)}\right)} .
$$

Proof of Theorem 1 is similar to Lemma 1 in [8]. Now, we continue to compute DE of $M=5, N=10$ and $M=6$, $N=10$. Tables 4 and 5 are the DE of five-layer and six-layer defense, respectively. Another useful rule is that the number of target channels deployed along the $i$ th layer should be not less than $(i+1)$ th layer; this rule is summarized in Theorem 2.

Theorem 2. Let $\mathbf{E}_{\left(\mathbf{M}, \mathbf{n}_{(M)}\right)}$ be the DE of the M-layer BMDS; $\mathbf{n}_{(\mathbf{M})}=\left(n_{1}, n_{2}, \ldots, n_{M}\right)$ is the allocation plan, $n_{i}$ is the number of target channels deployed along the ith layer, and $N=\sum_{i=1}^{M} n_{i}$, $n_{i} \geq 1$. For allocation plan $\mathbf{n}_{(\mathbf{M})}^{*}$, the number of target channels deployed along the ith layer is decreasing as i increases; that $i$, for all $i, n_{i} \geq n_{i+1}$. When $N$ is constant, then one has $\mathbf{E}_{\left(\mathbf{M}, \mathbf{n}_{(M)}^{*}\right)} \geq \mathbf{E}_{\left(\mathbf{M}, \mathbf{n}_{(M)}\right)}$.

Proof. Suppose we have a plan $\mathbf{n}_{(\mathbf{M})}^{\Theta}=\left(n_{1}, n_{2}, \ldots, n_{i}^{*}\right.$, $\left.n_{i+1}^{*}, \ldots, n_{M}\right)$, where $n_{i}^{*}=n_{i+1}, n_{i+1}^{*}=n_{i}$. In order to prove Theorem 2, we only need to prove $\mathbf{E}_{\left(\mathbf{M}, \mathbf{n}_{(M)}^{*}\right)} \geq \mathbf{E}_{\left(\mathbf{M}, \mathbf{n}_{(M)}^{\Theta}\right)}$, which can be simplified by proving $\mathbf{E}_{\left(2, \mathbf{n}_{(2)}^{*}\right)} \geq \mathbf{E}_{\left(2, \mathbf{n}_{(2)}^{\Theta}\right)}$, where $\mathbf{n}_{(2)}^{*}=$ $\left(n_{i}, n_{i+1}\right), \mathbf{n}_{(2)}^{\Theta}=\left(n_{i}^{*}, n_{i+1}^{*}\right)$. For function

$$
f(\rho, n)=P_{\text {lost }}=\left(\frac{\rho^{n}}{n !}\right)\left(\sum_{k=0}^{n} \frac{\rho^{k}}{k !}\right)^{-1},
$$

when $\rho$ is constant, $f(\rho, n)$ decreases with the increase of $n$, and when $n$ is constant, $f(\rho, n)$ increases with the increase of $\rho[2,20]$. For proof simplified by letting $P_{k}=1$ for all defense weapons, then $f\left(\rho, n_{i}\right), f\left(\rho, n_{i+1}\right), f\left(\rho, n_{i}^{*}\right)$, and $f\left(\rho, n_{i+1}^{*}\right)$ are

$$
\begin{aligned}
f\left(\rho, n_{i}\right) & =\left(\frac{\rho^{n_{i}}}{n_{i} !}\right)\left(\sum_{k=0}^{n_{i}} \frac{\rho^{k}}{k !}\right)^{-1}, \\
f\left(\rho, n_{i+1}\right) & =\left(\frac{\rho_{*}^{n_{i+1}}}{n_{i+1} !}\right)\left(\sum_{k=0}^{n_{i+1}} \frac{\rho_{*}^{k}}{k !}\right)^{-1}, \\
f\left(\rho, n_{i}^{*}\right) & =\left(\frac{\rho^{n_{i+1}}}{n_{i+1} !}\right)\left(\sum_{k=0}^{n_{i+1}} \frac{\rho^{k}}{k !}\right)^{-1}, \\
f\left(\rho, n_{i+1}^{*}\right) & =\left(\frac{\rho_{\Theta}^{n_{i}}}{n_{i} !}\right)\left(\sum_{k=0}^{n_{i}} \frac{\rho_{\Theta}^{k}}{k !}\right)^{-1},
\end{aligned}
$$

respectively, where $\rho_{*}=f\left(\rho, n_{i}\right) \cdot \rho, \rho_{\Theta}=f\left(\rho, n_{i}^{*}\right) \cdot \rho$. DE of the two-layer defense are

$$
\begin{aligned}
& \mathbf{E}_{\left(2, \mathbf{n}_{(2)}^{*}\right)} \\
& =P_{k} \cdot\left(1-f\left(\rho, n_{i}\right)+f\left(\rho, n_{i}\right) \cdot\left(1-f\left(\rho, n_{i+1}\right)\right)\right) \\
& =1-f\left(\rho, n_{i}\right) f\left(\rho, n_{i+1}\right), \\
& \mathbf{E}_{\left(2, \mathbf{n}_{(2)}^{\Theta}\right)} \\
& =P_{k} \\
& \quad \cdot\left(1-f\left(\rho, n_{i}^{*}\right)+f\left(\rho, n_{i}^{*}\right) \cdot\left(1-f\left(\rho, n_{i+1}^{*}\right)\right)\right) \\
& =1-f\left(\rho, n_{i}^{*}\right) f\left(\rho, n_{i+1}^{*}\right) .
\end{aligned}
$$


TABLE 2: DE of three-layer defense.

\begin{tabular}{|c|c|c|c|c|c|c|}
\hline 1st-channel number & 1st-layer DE & 2nd-channel number & 2nd-layer DE & 3rd-channel number & 3rd-layer DE & Total DE \\
\hline 8 & $55.552 \%$ & 1 & $11.872 \%$ & 1 & $11.872 \%$ & $65.479 \%$ \\
\hline 7 & $53.256 \%$ & 2 & $22.176 \%$ & 1 & $11.872 \%$ & $67.941 \%$ \\
\hline 7 & $53.256 \%$ & 1 & $11.872 \%$ & 2 & $22.176 \%$ & $67.941 \%$ \\
\hline 6 & $50.344 \%$ & 2 & $22.176 \%$ & 2 & $22.176 \%$ & $69.925 \%$ \\
\hline 6 & $50.344 \%$ & 3 & $32.368 \%$ & 1 & $11.872 \%$ & $70.404 \%$ \\
\hline 6 & $50.344 \%$ & 1 & $11.872 \%$ & 3 & $32.368 \%$ & $70.404 \%$ \\
\hline 5 & $46.368 \%$ & 3 & $32.368 \%$ & 2 & $22.176 \%$ & $71.771 \%$ \\
\hline 5 & $46.368 \%$ & 2 & $22.176 \%$ & 3 & $32.368 \%$ & $71.771 \%$ \\
\hline 5 & $46.368 \%$ & 4 & $38.264 \%$ & 1 & $11.872 \%$ & $70.821 \%$ \\
\hline 5 & $46.368 \%$ & 1 & $11.872 \%$ & 4 & $38.264 \%$ & $70.821 \%$ \\
\hline 4 & $38.264 \%$ & 3 & $32.368 \%$ & 3 & $32.368 \%$ & $71.761 \%$ \\
\hline 4 & $38.264 \%$ & 4 & $38.264 \%$ & 2 & $22.176 \%$ & $70.339 \%$ \\
\hline 4 & $38.264 \%$ & 2 & $22.176 \%$ & 4 & $38.264 \%$ & $70.339 \%$ \\
\hline 4 & $38.264 \%$ & 5 & $46.368 \%$ & 1 & $11.872 \%$ & $70.821 \%$ \\
\hline 4 & $38.264 \%$ & 1 & $11.872 \%$ & 5 & $46.368 \%$ & $70.821 \%$ \\
\hline 3 & $32.368 \%$ & 4 & $38.264 \%$ & 3 & $32.368 \%$ & $71.761 \%$ \\
\hline 3 & $32.368 \%$ & 3 & $32.368 \%$ & 4 & $38.264 \%$ & $71.761 \%$ \\
\hline 3 & $32.368 \%$ & 5 & $46.368 \%$ & 2 & $22.176 \%$ & $71.771 \%$ \\
\hline 3 & $32.368 \%$ & 2 & $22.176 \%$ & 5 & $46.368 \%$ & $71.771 \%$ \\
\hline 3 & $32.368 \%$ & 6 & $50.344 \%$ & 1 & $11.872 \%$ & $70.404 \%$ \\
\hline 3 & $32.368 \%$ & 1 & $11.872 \%$ & 6 & $50.344 \%$ & $70.404 \%$ \\
\hline 2 & $22.176 \%$ & 4 & $38.264 \%$ & 4 & $38.264 \%$ & $70.339 \%$ \\
\hline 2 & $22.176 \%$ & 5 & $46.368 \%$ & 3 & $32.368 \%$ & $71.771 \%$ \\
\hline 2 & $22.176 \%$ & 3 & $32.368 \%$ & 5 & $46.368 \%$ & $71.771 \%$ \\
\hline 2 & $22.176 \%$ & 6 & $50.344 \%$ & 2 & $22.176 \%$ & $69.925 \%$ \\
\hline 2 & $22.176 \%$ & 2 & $22.176 \%$ & 6 & $50.344 \%$ & $69.925 \%$ \\
\hline 2 & $22.176 \%$ & 7 & $53.256 \%$ & 1 & $11.872 \%$ & $67.941 \%$ \\
\hline 2 & $22.176 \%$ & 1 & $11.872 \%$ & 7 & $53.256 \%$ & $67.941 \%$ \\
\hline 1 & $11.872 \%$ & 5 & $46.368 \%$ & 4 & $38.264 \%$ & $70.821 \%$ \\
\hline 1 & $11.872 \%$ & 4 & $38.264 \%$ & 5 & $46.368 \%$ & $70.821 \%$ \\
\hline 1 & $11.872 \%$ & 6 & $50.344 \%$ & 3 & $32.368 \%$ & $70.404 \%$ \\
\hline 1 & $11.872 \%$ & 3 & $32.368 \%$ & 6 & $50.344 \%$ & $70.404 \%$ \\
\hline 1 & $11.872 \%$ & 7 & $53.256 \%$ & 2 & $22.176 \%$ & $67.941 \%$ \\
\hline 1 & $11.872 \%$ & 2 & $22.176 \%$ & 7 & $53.256 \%$ & $67.941 \%$ \\
\hline 1 & $11.872 \%$ & 8 & $55.552 \%$ & 1 & $11.872 \%$ & $65.479 \%$ \\
\hline 1 & $11.872 \%$ & 1 & $11.872 \%$ & 8 & $55.552 \%$ & $65.479 \%$ \\
\hline
\end{tabular}

Since the expansion of $e^{x}$ is $1+x+x^{2} / 2 !+\cdots+x^{n} / n$ !, then the approximate representations of $f\left(\rho, n_{i}\right), f\left(\rho, n_{i+1}\right), f\left(\rho, n_{i}^{*}\right)$, and $f\left(\rho, n_{i+1}^{*}\right)$ are

$$
\begin{aligned}
f\left(\rho, n_{i}\right) & \approx\left(\frac{\rho^{n_{i}}}{n_{i} !}\right)\left(e^{\rho}\right)^{-1}, \\
f\left(\rho, n_{i+1}\right) & \approx\left(\frac{\rho_{*}^{n_{i+1}}}{n_{i+1} !}\right)\left(e^{\rho_{*}}\right)^{-1}, \\
f\left(\rho, n_{i}^{*}\right) & \approx\left(\frac{\rho^{n_{i+1}}}{n_{i+1} !}\right)\left(e^{\rho}\right)^{-1}, \\
f\left(\rho, n_{i+1}^{*}\right) & =\left(\frac{\rho_{\Theta}^{n_{i}}}{n_{i} !}\right)\left(e^{\rho_{\Theta}}\right)^{-1},
\end{aligned}
$$

respectively. Because $n_{i} \geq n_{i+1}$, we have

$$
\begin{aligned}
& f\left(\rho, n_{i}^{*}\right) \geq f\left(\rho, n_{i}\right), \\
& f\left(\rho, n_{i}^{*}\right) \geq f\left(\rho, n_{i+1}\right), \\
& f\left(\rho, n_{i}\right) \geq f\left(\rho, n_{i+1}^{*}\right) .
\end{aligned}
$$

In order to prove Theorem 2, we need to prove

$$
\begin{aligned}
& 1-f\left(\rho, n_{i}\right) f\left(\rho, n_{i+1}\right) \geq 1-f\left(\rho, n_{i}^{*}\right) f\left(\rho, n_{i+1}^{*}\right) \Longleftarrow \\
& f\left(\rho, n_{i}^{*}\right) f\left(\rho, n_{i+1}^{*}\right)-f\left(\rho, n_{i}\right) f\left(\rho, n_{i+1}\right) \geq 0 \Longleftarrow
\end{aligned}
$$


TABLE 3: DE of four-layer defense.

\begin{tabular}{|c|c|c|c|c|c|c|c|c|}
\hline $\begin{array}{l}\text { 1st-channel } \\
\text { number }\end{array}$ & 1st-layer DE & $\begin{array}{l}\text { 2nd- } \\
\text { channel } \\
\text { number }\end{array}$ & 2nd-layer DE & $\begin{array}{l}\text { 3rd-channel } \\
\text { number }\end{array}$ & 3rd-layer DE & $\begin{array}{c}\text { 4th-channel } \\
\text { number }\end{array}$ & 4th-layer DE & Total DE \\
\hline 7 & $53.200 \%$ & 1 & $11.872 \%$ & 1 & $11.872 \%$ & 1 & $11.872 \%$ & $67.968 \%$ \\
\hline 6 & $50.344 \%$ & 2 & $17.360 \%$ & 1 & $11.872 \%$ & 1 & $11.872 \%$ & $68.129 \%$ \\
\hline 6 & $50.344 \%$ & 1 & $11.872 \%$ & 2 & $17.360 \%$ & 1 & $11.872 \%$ & $68.129 \%$ \\
\hline 5 & $47.600 \%$ & 3 & $34.920 \%$ & 1 & $11.872 \%$ & 1 & $11.872 \%$ & $73.515 \%$ \\
\hline 5 & $47.600 \%$ & 2 & $17.360 \%$ & 2 & $17.360 \%$ & 1 & $11.872 \%$ & $68.463 \%$ \\
\hline 5 & $47.600 \%$ & 1 & $11.872 \%$ & 3 & $34.920 \%$ & 1 & $11.872 \%$ & $73.515 \%$ \\
\hline 4 & $45.920 \%$ & 3 & $34.920 \%$ & 2 & $17.360 \%$ & 1 & $11.872 \%$ & $74.368 \%$ \\
\hline 4 & $45.920 \%$ & 3 & $34.920 \%$ & 1 & $11.872 \%$ & 2 & $17.360 \%$ & $74.368 \%$ \\
\hline 4 & $45.920 \%$ & 2 & $17.360 \%$ & 1 & $11.872 \%$ & 3 & $34.920 \%$ & $74.368 \%$ \\
\hline 4 & $45.920 \%$ & 2 & $17.360 \%$ & 3 & $34.920 \%$ & 1 & $11.872 \%$ & $74.368 \%$ \\
\hline 4 & $45.920 \%$ & 1 & $11.872 \%$ & 2 & $17.360 \%$ & 3 & $34.920 \%$ & $74.368 \%$ \\
\hline 4 & $45.920 \%$ & 1 & $11.872 \%$ & 3 & $34.920 \%$ & 2 & $17.360 \%$ & $74.368 \%$ \\
\hline 3 & $34.920 \%$ & 5 & $47.600 \%$ & 1 & $11.872 \%$ & 1 & $11.872 \%$ & $73.515 \%$ \\
\hline 3 & $34.920 \%$ & 4 & $45.920 \%$ & 2 & $17.360 \%$ & 1 & $11.872 \%$ & $74.368 \%$ \\
\hline 3 & $34.920 \%$ & 4 & $45.920 \%$ & 1 & $11.872 \%$ & 2 & $17.360 \%$ & $74.368 \%$ \\
\hline 3 & $34.920 \%$ & 3 & $34.920 \%$ & 2 & $17.360 \%$ & 2 & $17.360 \%$ & $71.075 \%$ \\
\hline 3 & $34.920 \%$ & 2 & $17.360 \%$ & 3 & $34.920 \%$ & 2 & $17.360 \%$ & $71.075 \%$ \\
\hline 3 & $34.920 \%$ & 2 & $17.360 \%$ & 2 & $17.360 \%$ & 3 & $34.920 \%$ & $71.075 \%$ \\
\hline 3 & $34.920 \%$ & 2 & $17.360 \%$ & 4 & $45.920 \%$ & 1 & $11.872 \%$ & $74.368 \%$ \\
\hline 3 & $34.920 \%$ & 2 & $17.360 \%$ & 1 & $11.872 \%$ & 4 & $45.920 \%$ & $74.368 \%$ \\
\hline 3 & $34.920 \%$ & 1 & $11.872 \%$ & 4 & $45.920 \%$ & 2 & $17.360 \%$ & $74.368 \%$ \\
\hline 3 & $34.920 \%$ & 1 & $11.872 \%$ & 2 & $17.360 \%$ & 4 & $45.920 \%$ & $74.368 \%$ \\
\hline 3 & $34.920 \%$ & 1 & $11.872 \%$ & 5 & $47.600 \%$ & 1 & $11.872 \%$ & $73.515 \%$ \\
\hline 2 & $17.360 \%$ & 6 & $50.344 \%$ & 1 & $11.872 \%$ & 1 & $11.872 \%$ & $68.129 \%$ \\
\hline 2 & $17.360 \%$ & 5 & $47.600 \%$ & 2 & $17.360 \%$ & 1 & $11.872 \%$ & $68.463 \%$ \\
\hline 2 & $17.360 \%$ & 4 & $45.920 \%$ & 3 & $34.920 \%$ & 3 & $34.920 \%$ & $71.071 \%$ \\
\hline 2 & $17.360 \%$ & 3 & $34.920 \%$ & 3 & $34.920 \%$ & 2 & $17.360 \%$ & $71.075 \%$ \\
\hline 2 & $17.360 \%$ & 3 & $34.920 \%$ & 2 & $17.360 \%$ & 3 & $34.920 \%$ & $71.075 \%$ \\
\hline 1 & $11.872 \%$ & 7 & $53.200 \%$ & 1 & $11.872 \%$ & 1 & $11.872 \%$ & $67.968 \%$ \\
\hline 1 & $11.872 \%$ & 6 & $50.344 \%$ & 2 & $17.360 \%$ & 1 & $11.872 \%$ & $68.129 \%$ \\
\hline
\end{tabular}

$$
\begin{aligned}
& \frac{f\left(\rho, n_{i}^{*}\right) f\left(\rho, n_{i+1}^{*}\right)}{f\left(\rho, n_{i}\right) f\left(\rho, n_{i+1}\right)} \geq 1, \\
& \frac{f\left(\rho, n_{i}^{*}\right) f\left(\rho, n_{i+1}^{*}\right)}{f\left(\rho, n_{i}\right) f\left(\rho, n_{i+1}\right)} \\
& =\frac{\left(\left(\rho^{n_{i+1}} / n_{i+1} !\right)\left(e^{\rho}\right)^{-1}\right)\left(\left(\rho_{\Theta}^{n_{i}} / n_{i} !\right)\left(e^{\rho_{\Theta}}\right)^{-1}\right)}{\left(\left(\rho^{n_{i}} / n_{i} !\right)\left(e^{\rho}\right)^{-1}\right)\left(\left(\rho_{*}^{n_{i+1}} / n_{i+1} !\right)\left(e^{\rho_{*}}\right)^{-1}\right)} \\
& =\frac{\rho^{n_{i+1}} \rho_{\Theta}^{n_{i}} e^{\rho_{*}}}{\rho^{n_{i}} \rho_{*}^{n_{i+1}} e^{\rho_{\Theta}}}=\frac{\rho^{n_{i+1}} \rho_{\Theta}^{n_{i}} e^{f\left(\rho, n_{i}\right) \cdot \rho}}{\rho^{n_{i}} \rho_{*}^{n_{i+1}} e^{f\left(\rho, n_{i}^{*}\right) \cdot \rho}} \\
& =\frac{\rho^{n_{i+1}}\left(f\left(\rho, n_{i}^{*}\right) \cdot \rho\right)^{n_{i}} e^{f\left(\rho, n_{i}\right) \cdot \rho}}{\rho^{n_{i}}\left(f\left(\rho, n_{i}\right) \cdot \rho\right)^{n_{i+1}} e^{f\left(\rho, n_{i}^{*}\right) \cdot \rho}}
\end{aligned}
$$$$
=\frac{\left(f\left(\rho, n_{i}^{*}\right)\right)^{n_{i}} e^{f\left(\rho, n_{i}\right) \cdot \rho}}{\left(f\left(\rho, n_{i}\right)\right)^{n_{i+1}} e^{f\left(\rho, n_{i}^{*}\right) \cdot \rho}}=\frac{\left(n_{i} !\right)^{n_{i+1}} e^{n_{i+1} \cdot \rho} e^{f\left(\rho, n_{i}\right) \cdot \rho}}{\left(n_{i+1} !\right)^{n_{i}} e^{n_{i} \cdot \rho} e^{f\left(\rho, n_{i}^{*}\right) \cdot \rho}}
$$$$
=\frac{\left(n_{i} !\right)^{n_{i+1}}}{\left(n_{i+1} !\right)^{n_{i}}} e^{\left(n_{i+1}+f\left(\rho, n_{i}\right)-n_{i}-f\left(\rho, n_{i}^{*}\right)\right) \cdot \rho} .
$$

When $n_{i}=n_{i+1}$, Theorem 2 is true. Obviously, when $n_{i}>n_{i+1}$, we have $\left(n_{i} !\right)^{n_{i+1}} \gg\left(n_{i+1} !\right)^{n_{i}}$; then, $\mathbf{E}_{\left(2, \mathbf{n}_{(2)}^{*}\right)}>\mathbf{E}_{\left(2, \mathbf{n}_{(2)}^{\Theta}\right)}$, and the result of Theorem 2 follows.

4.1.2. Minimum Requirements of Target Channels. The requirements of target channels necessary to achieve a demanded DE can be viewed as a dual problem of (41). 
TABLE 4: DE of five-layer defense.

\begin{tabular}{lcccccccccc}
\hline 1st number & 1st DE & 2nd number & 2nd DE & 3rd number & 3rd DE & 4th number & 4th DE & 5th number & 5th DE & Total DE \\
\hline 5 & $45.5 \%$ & 2 & $23.4 \%$ & 1 & $12.0 \%$ & 1 & $12.0 \%$ & 1 & $12.0 \%$ & $71.5 \%$ \\
4 & $38.6 \%$ & 3 & $30.8 \%$ & 1 & $12.0 \%$ & 1 & $12.0 \%$ & 1 & $12.0 \%$ & $71.0 \%$ \\
4 & $38.6 \%$ & 2 & $23.4 \%$ & 2 & $23.4 \%$ & 1 & $12.0 \%$ & 1 & $12.0 \%$ & $72.0 \%$ \\
3 & $30.8 \%$ & 3 & $30.8 \%$ & 2 & $23.4 \%$ & 1 & $12.0 \%$ & 1 & $12.0 \%$ & $71.6 \%$ \\
$\mathbf{3}$ & $\mathbf{3 0 . 8} \%$ & $\mathbf{3}$ & $\mathbf{3 0 . 8} \%$ & $\mathbf{2}$ & $\mathbf{2 3 . 4 \%}$ & $\mathbf{1}$ & $\mathbf{1 2 . 0} \%$ & $\mathbf{1}$ & $\mathbf{1 2 . 0 \%}$ & $\mathbf{7 4 . 6 \%}$ \\
2 & $23.4 \%$ & 2 & $23.4 \%$ & 2 & $23.4 \%$ & 2 & $23.4 \%$ & 2 & $23.4 \%$ & $\mathbf{7 3 . 5 \%}$ \\
\hline
\end{tabular}

TABLE 5: DE of six-layer defense.

\begin{tabular}{lcccccccccccc}
\hline 1st number & 1st DE & 2nd number & 2nd DE & 3rd number & 3rd DE & 4th number & 4th DE & 5th number & 5th DE & 6th number & 6th DE & Total DE \\
\hline 5 & $45 \%$ & 1 & $14 \%$ & 1 & $14 \%$ & 1 & $14 \%$ & 1 & $14 \%$ & 1 & $14 \%$ & $74 \%$ \\
4 & $39 \%$ & 2 & $26 \%$ & 1 & $14 \%$ & 1 & $14 \%$ & 1 & $14 \%$ & 1 & $14 \%$ & $75 \%$ \\
$\mathbf{3}$ & $\mathbf{4 1 \%}$ & $\mathbf{2}$ & $\mathbf{2 6 \%}$ & $\mathbf{2}$ & $\mathbf{2 6 \%}$ & $\mathbf{1}$ & $\mathbf{1 4 \%}$ & $\mathbf{1}$ & $\mathbf{1 4 \%}$ & $\mathbf{1}$ & $\mathbf{1 4 \%}$ & $\mathbf{7 9 \%}$ \\
2 & $26 \%$ & 3 & $41 \%$ & 2 & $26 \%$ & 1 & $14 \%$ & 1 & $14 \%$ & 1 & $14 \%$ & $79 \%$ \\
2 & $26 \%$ & 2 & $26 \%$ & 2 & $26 \%$ & 2 & $26 \%$ & 1 & $14 \%$ & 1 & $14 \%$ & $77 \%$ \\
\hline
\end{tabular}

Assuming that the $\mathrm{DE}$ is held at greater than $E^{*}$, we define the optimization problem so as to minimize the requirements of target channels, subject to a given set of constraints:

$$
\begin{array}{ll}
\min \quad n=\sum_{i=1}^{M} n_{i} \\
\text { s.t. } \quad \rho_{i} \\
=\left(1-E_{i-1}\right)\left(1-E_{i-2}\right) \cdots\left(1-E_{1}\right) \lambda \cdot t_{w \text { mean }} \\
\quad \cdot P_{f}=\left(\prod_{x=1}^{i-1}\left(1-E_{i}\right)\right) \cdot \rho_{1} \\
=\left(1-E_{i-1}\right) \cdot \rho_{i-1}, \\
P_{\text {shoot }}^{(i)}=1-\left(\frac{\rho_{i}^{n_{i}}}{n_{i} !}\right)\left(\sum_{k=0}^{n_{i}} \frac{\rho_{i}^{k}}{k !}\right)^{-1}, \\
E^{*} \\
\leq & E_{1}+\left(1-E_{1}\right) E_{2}+\cdots+\prod_{i=1}^{M-1}\left(1-E_{i}\right) E_{M}, \\
& E_{i}=P_{\text {shoot }}^{(i)} \cdot P_{k} .
\end{array}
$$

For the nonlinear optimization problem (49), when problem size is small, we can use algebra, dynamic programming, or enumeration method to solve it. When the size of the problem is very large, an approximate solution can be obtained by using some advanced algorithms, for example, genetic and heuristic algorithms. Then, we give the definition of neighborhood [21].

Definition 3. Let $\mathbf{n}_{(\mathbf{M})}=\left(n_{1}, n_{2}, \ldots, n_{M}\right) \in \Omega$ be an allocation plan, and $\Omega$ is the feasible region of allocation plans. Suppose that $\mathbf{n}_{(\mathbf{M})}^{*}=\left(n_{1}, n_{2}, \ldots, n_{i-1}, n_{i}-1, n_{i+1}, \ldots, n_{M}\right) \in \Omega$, where the number of target channels deployed along the $i$ th layer is $n_{i}-1$. Then, one says that $\mathbf{n}_{(\mathbf{M})}^{*}$ is in the neighborhood of $\mathbf{n}_{(\mathbf{M})}$; that is,

$$
\begin{aligned}
& \left(n_{1}, n_{2}, \ldots, n_{i-1}, n_{i}-1, n_{i+1}, \ldots, n_{M}\right) \\
& \quad \in \varphi\left(n_{1}, n_{2}, \ldots, n_{M}\right) .
\end{aligned}
$$

Scenario 2. Suppose the SSKP is 0.7 , the probability that BMs will be detected by radars is $0.8, \lambda=5 \mathrm{BMs} / \mathrm{min}, t_{\text {smean }}=$ $0.75 \mathrm{~min}$, and $E^{*}=65 \%$; then the question becomes as follows: "What is the least cost of target channels to achieve a demanded DE?"

Figure 4 is the schematic of search in the neighborhood of $\mathbf{n}_{(3)}=(5,3,2)$ and $\mathbf{n}_{(4)}=(4,3,2,1)$. We can see the least cost is 9 channels for three-layer defense and 8 channels for four-layer defense, and allocation plans are $\mathbf{n}_{(3)}=(4,3,2)$ and $\mathbf{n}_{(4)}=(3,3,1,1)$, respectively. So, we give a simple algorithm in finding the least cost of DWs to achieve a demanded DE; specific search methods are as follows. A feasible initial allocation plan is very important in this algorithm. Theorem 1 proposes the basic rule of finding an initial plan, which greatly simplifies the searching process.

Step 1. Suppose the number of layers is $M$; the demanded $\mathrm{DE}$ is $E^{*}$; and give an initial allocation plan $\mathbf{n}_{(\mathbf{M})}=$ $\left(n_{1}, n_{2}, \ldots, n_{M}\right) \in \Omega$, where $\mathbf{E}_{\left(\mathbf{M}, \mathbf{n}_{(M)}\right)} \geq E^{*}$.

Step 2. Search in the neighborhood of $\mathbf{n}_{(\mathbf{M})}, \varphi\left(\mathbf{n}_{(\mathbf{M})}\right)$, and try to find an allocation plan $\varphi_{1}\left(\mathbf{n}_{(\mathbf{M})}\right)$ satisfying the following:

$$
\mathbf{E}_{\left(\mathbf{M}, \varphi_{1}\left(\mathbf{n}_{(M)}\right)\right)}=\max \mathbf{E}_{\left(\mathbf{M}, \varphi\left(\mathbf{n}_{(M)}\right)\right)} \text {. }
$$

Step 3. If $\mathbf{E}_{\left(\mathbf{M}, \varphi_{1}\left(\mathbf{n}_{(M)}\right)\right)} \geq E^{*}$, then $\mathbf{n}_{(\mathbf{M})}=\varphi_{1}\left(\mathbf{n}_{(\mathbf{M})}\right)$; go to Step 2 and repeat.

Step 4. If $\mathbf{E}_{\left(\mathbf{M}, \varphi_{1}\left(\mathbf{n}_{(\mathbf{M})}\right)\right)}<E^{*}$, go to Step 5 .

Step 5. Output the allocation plan $\mathbf{n}_{(\mathbf{M})} ; \sum_{i=1}^{M} n_{i}$ is the least cost to achieve the demanded DE. 


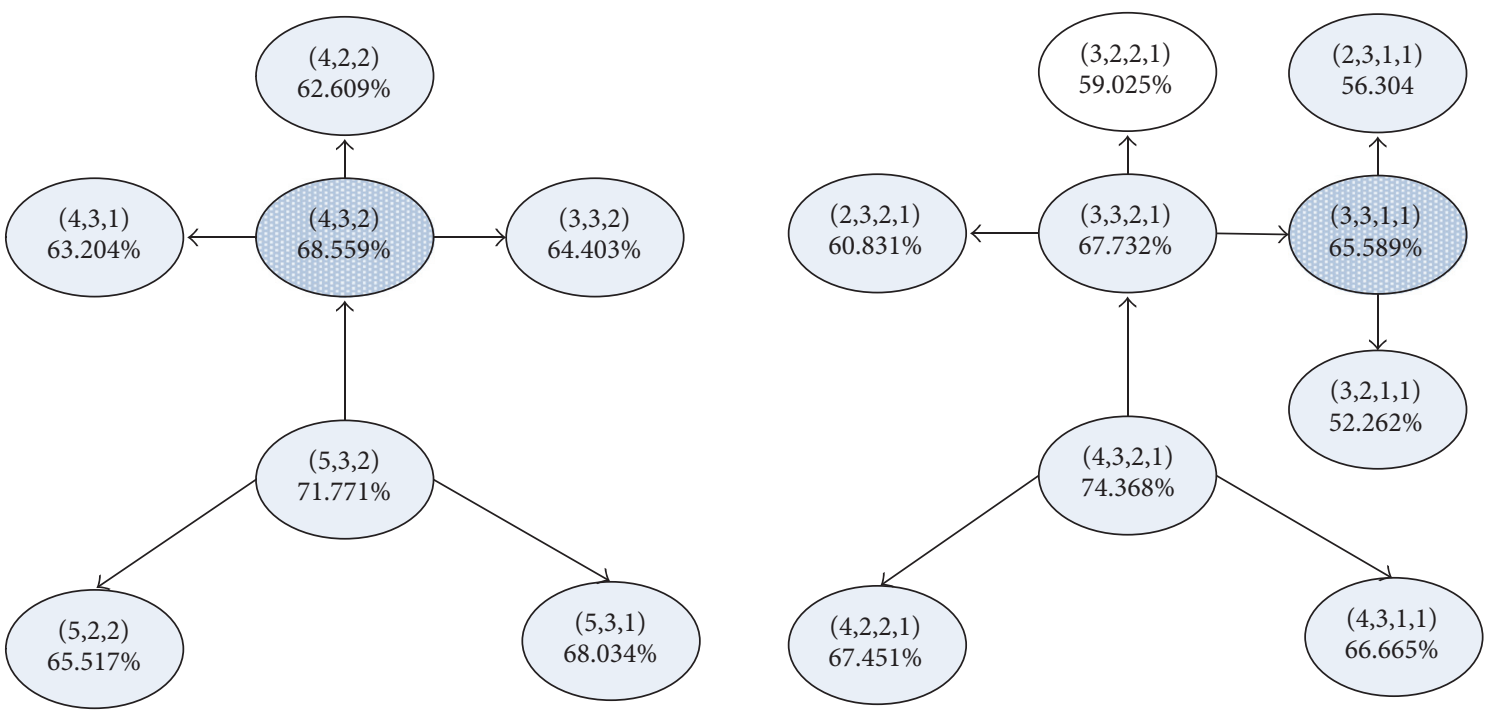

FIGURE 4: Search in the neighborhood of $\mathbf{n}_{(3)}=(5,3,2)$ and $\mathbf{n}_{(4)}=(4,3,2,1)$.

\subsection{Different Defense Weapons and $M / M / N / N$ System}

4.2.1. Different Defense Weapons and Identical SSKPs. In this section, we consider different types of defense weapons. Let $M$ be the number of layers; the number of target channels deployed along the $i$ th layer will be denoted by $n_{i}$, assuming that the total number of types is $M$ and defense weapons are identical in the same layer. As in Section 3.3, subscript $(\cdot)_{(i)}$ indicates defense weapons of type $i$; then probabilities that BMs will be shot by the $M$-layer defense with short defense depth are as follows:

1st layer:

$$
\begin{aligned}
\rho_{1(1)} & =\lambda \cdot t_{w \operatorname{mean}(1)} \cdot P_{f(1)}, \\
P_{\text {shoot }}^{(1)} & =1-\left(\frac{\rho_{1(1)}^{n_{1}}}{n_{1} !}\right)\left(\sum_{k=0}^{n_{1}} \frac{\rho_{1(1)}^{k}}{k !}\right)^{-1}, \\
E_{1} & =P_{\text {shoot }}^{(1)} \cdot P_{k(1)} .
\end{aligned}
$$

2nd layer:

$$
\begin{aligned}
\rho_{2(2)} & =\left(1-E_{1}\right) \cdot \lambda \cdot t_{w \operatorname{mean}(2)} \cdot P_{f(2)}, \\
P_{\text {shoot }}^{(2)} & =1-\left(\frac{\rho_{2(2)}^{n_{2}}}{n_{2} !}\right)\left(\sum_{k=0}^{n_{2}} \frac{\rho_{2(2)}^{k}}{k !}\right)^{-1}, \\
E_{2} & =P_{\text {shoot }}^{(2)} \cdot P_{k(2)} .
\end{aligned}
$$

Mth layer:

$$
\begin{aligned}
\rho_{M(M)}= & \left(1-E_{M-1}\right)\left(1-E_{M-2}\right) \cdots\left(1-E_{1}\right) \lambda \\
& \cdot t_{w \operatorname{mean}(M)} \cdot P_{f(M)}
\end{aligned}
$$

$$
\begin{aligned}
& =\left(\prod_{i=1}^{M-1}\left(1-E_{i}\right)\right) \cdot \lambda \cdot t_{\text {wean }(M)} \cdot P_{f(M)}, \\
P_{\text {shoot }}^{(M)} & =1-\left(\frac{\rho_{M(M)}^{n_{M}}}{n_{M} !}\right)\left(\sum_{k=0}^{n_{M}} \frac{\rho_{M(M)}^{k}}{k !}\right)^{-1}, \\
E_{M} & =P_{\text {shoot }}^{(M)} \cdot P_{k(M)} .
\end{aligned}
$$

$\mathrm{DE}$ of the whole $M$-layer BMDS is

$$
\begin{aligned}
E= & E_{1}+\left(1-E_{1}\right) E_{2}+\left(1-E_{1}\right)\left(1-E_{2}\right) E_{3} \cdots \\
& +\prod_{i=1}^{M-1}\left(1-E_{i}\right) E_{M} \\
= & E_{1}+\sum_{k=2}^{M}\left(E_{k} \cdot \prod_{i=1}^{M-1}\left(1-E_{i}\right)\right) .
\end{aligned}
$$

For certain types and numbers of target channels, we define the optimization problem as finding which type of defense weapon should be deployed on each layer so as to maximize the ED, subject to a given set of constraints:

$\max E$

$$
\begin{aligned}
= & E_{1}+\left(1-E_{1}\right) E_{2}+\left(1-E_{1}\right)\left(1-E_{2}\right) E_{3} \cdots \\
& +\prod_{i=1}^{M-1}\left(1-E_{i}\right) E_{M}
\end{aligned}
$$


TABLE 6: DE of three-layer defense.

\begin{tabular}{|c|c|c|c|c|c|c|}
\hline 1st-layer type & 1st-layer DE & 2nd-layer type & 2nd-layer DE & 3rd-layer type & 3rd-layer DE & Total DE \\
\hline II & $43.200 \%$ & II & $45.440 \%$ & I & $55.520 \%$ & $86.216 \%$ \\
\hline II & $48.960 \%$ & I & $51.840 \%$ & II & $46.560 \%$ & $86.864 \%$ \\
\hline II & $43.200 \%$ & I & $55.040 \%$ & I & $55.040 \%$ & $88.518 \%$ \\
\hline I & $54.560 \%$ & II & $45.280 \%$ & II & $46.720 \%$ & $86.752 \%$ \\
\hline I & $56.800 \%$ & I & $55.360 \%$ & II & $43.040 \%$ & $89.016 \%$ \\
\hline
\end{tabular}

s.t. $\rho_{i(M)}$

$$
\begin{aligned}
&=\left(1-E_{i-1}\right)\left(1-E_{i-2}\right) \cdots\left(1-E_{1}\right) \lambda \\
& \cdot t_{w \operatorname{mean}(M)} \cdot P_{f(M)} \\
&=\left(\prod_{x=1}^{i-1}\left(1-E_{i}\right)\right) \cdot \lambda \cdot t_{w \operatorname{mean}(M)} \cdot P_{f(M)}, \\
& P_{\text {shoot }}^{(i)}=1-\left(\frac{\rho_{i(M)}^{n_{i}}}{n_{i} !}\right)\left(\sum_{k=0}^{n_{i}} \frac{\rho_{i(M)}^{k}}{k !}\right)^{-1} \\
& E_{i}=P_{\text {shoot }}^{(i)} \cdot P_{k(M)}, \\
& n=\sum_{i=1}^{M} n_{i} .
\end{aligned}
$$

In order to get some potential and useful allocation rules, we analyze a scenario.

Scenario 3. Suppose that the number of layers is 3 , the number of defense weapon types is 2 (types I and II), $P_{k(\mathrm{I})}=$ $P_{k(\mathrm{II})}=0.7$, the probability that BMs will be detected by radars is $0.8, \lambda=5 \mathrm{BMs} / \mathrm{min}, t_{s m e a n(\mathrm{I})}=0.75 \mathrm{~min}$, and $t_{\text {smean(II) }}=1 \mathrm{~min}$.

Table 6 is the DE of three-layer defense.

It can be seen from Table 6 that plan $\mathbf{n}_{(3)}=($ I, I, II) has the biggest DE. We also found that $\mathbf{n}_{(4)}=(\mathrm{I}, \mathrm{I}, \mathrm{I}, \mathrm{II})$ is the best, and then we have Theorem 4 .

Theorem 4. Let $\mathbf{E}_{\left(\mathbf{M}, \mathbf{n}_{(M)}\right)}$ be the DE of the M-layer BMDS; $\mathbf{n}_{(\mathbf{M})}=\left(n_{1}, n_{2}, \ldots, n_{M}\right)$ is the allocation plan, $n_{i}$ is the number of target channels deployed along the ith layer, and let $n_{1}=n_{2}=$ $\cdots=n_{M}$. Allocation plan $\mathbf{n}_{(\mathbf{M})}^{I}=(I, I I)$ indicates that defense weapons of type I are forward-deployed, and $\mathbf{n}_{(\mathbf{M})}^{I I}=(I I, I)$ indicates that defense weapons of type II are forward-deployed. Suppose that defense weapons are identical in the same layer, and $P_{k(I)}=P_{k(I I)}$ and $P_{f(I)}=P_{f(I I)}$; ift $t_{\text {smean }(I)} \leq t_{\text {smean }(I I)}$, then one has $\mathbf{E}_{\left(\mathbf{M}, \mathbf{n}_{(\mathbf{M})}^{I}\right)} \geq \mathbf{E}_{\left(\mathbf{M}, \mathbf{n}_{(\mathbf{M})}^{I I}\right)}$.

Proof. Suppose that we have an allocation plan $\mathbf{n}_{(\mathbf{M})}^{\mathrm{I}^{\Theta}}=$ $\left(\mathrm{I}^{\Theta}, \mathrm{II}^{\Theta}\right)$, where $t_{\text {smean }\left(\mathrm{I}^{\Theta}\right)}=t_{\text {smean(II) }}$ and $t_{\text {smean }\left(\mathrm{II}^{\Theta}\right)}=t_{\text {smean }(\mathrm{I})}$.
In order to prove Theorem 4 , we only need to prove $\mathbf{E}_{\left(\mathbf{M}, \mathbf{n}_{(\mathbf{M})}^{\mathrm{I}}\right)} \geq$ $\mathbf{E}_{\left(\mathbf{M}, \mathbf{n}_{(\mathbf{M})}^{\Theta}\right)}$. For function

$$
f(\rho, n)=P_{\text {lost }}=\left(\frac{\rho^{n}}{n !}\right)\left(\sum_{k=0}^{n} \frac{\rho^{k}}{k !}\right)^{-1},
$$

when $\rho$ is constant, $f(\rho, n)$ decreases with the increase of $n$, and when $n$ is constant, $f(\rho, n)$ increases with the increase of $\rho[2,20]$. Then, $f(\rho, \mathrm{I}), f(\rho, \mathrm{II}), f\left(\rho, \mathrm{I}^{\Theta}\right)$, and $f\left(\rho, \mathrm{II}^{\Theta}\right)$ are

$$
\begin{gathered}
f(\rho, \mathrm{I})=\left(\frac{\rho_{(\mathrm{I})}^{n}}{n !}\right)\left(\sum_{k=0}^{n} \frac{\rho_{(\mathrm{I})}^{k}}{k !}\right)^{-1}, \\
f(\rho, \mathrm{II})=\left(\frac{\rho_{(\mathrm{II})}^{n}}{n !}\right)\left(\sum_{k=0}^{n} \frac{\rho_{(\mathrm{II})}^{k}}{k !}\right)^{-1}, \\
f\left(\rho, \mathrm{I}^{\Theta}\right)=\left(\frac{\rho_{\left(\mathrm{I}^{\Theta}\right)}^{n}}{n !}\right)\left(\sum_{k=0}^{n} \frac{\rho_{\left(\mathrm{I}^{\Theta}\right)}^{k}}{k !}\right)^{-1}, \\
f\left(\rho, \mathrm{II}^{\Theta}\right)=\left(\frac{\rho_{\left(\mathrm{II} \mathrm{I}^{\Theta}\right)}^{n}}{n !}\right)\left(\sum_{k=0}^{n} \frac{\left.\rho_{(\mathrm{II}}^{k}\right)}{k !}\right)^{-1},
\end{gathered}
$$

where

$$
\begin{aligned}
& \rho_{(\mathrm{I})}=\lambda \cdot t_{s \operatorname{mean}(\mathrm{I})} \cdot P_{f(\mathrm{I})}, \\
& \rho_{\left(\mathrm{I}^{\Theta}\right)}=\lambda \cdot t_{s \operatorname{mean}\left(\mathrm{I}^{\Theta}\right)} \cdot P_{f\left(\mathrm{I}^{\Theta}\right)}, \\
& \rho_{(\mathrm{II})}=\left(1-E_{(\mathrm{I})}\right) \lambda t_{s m e a n(\mathrm{II})} P_{f(\mathrm{II})}, \\
& \rho_{\left(\mathrm{II}^{\Theta}\right)}\left.=\left(1-E_{\left(\mathrm{I}^{\Theta}\right)}\right) \lambda t_{\text {smean }(\mathrm{II}}{ }^{\Theta}\right) \\
& E_{f\left(\mathrm{I}^{\Theta}\right)}, \\
& E_{(\mathrm{I})}=1-f(\rho, \mathrm{I}), \\
& E_{\left(\mathrm{I}^{\Theta}\right)}=1-f\left(\rho, \mathrm{I}^{\Theta}\right) .
\end{aligned}
$$


DE of the defense are

$$
\begin{aligned}
& \mathbf{E}_{\left(\mathbf{M}, \mathbf{n}_{(\mathrm{M})}^{\mathrm{I}}\right)} \\
& =P_{k} \cdot(1-f(\rho, \mathrm{I})+f(\rho, \mathrm{I}) \cdot(1-f(\rho, \mathrm{II}))) \\
& =1-f(\rho, \mathrm{I}) f(\rho, \mathrm{II}), \\
& \mathbf{E}_{\left(\mathbf{M}, \mathbf{n}_{(\mathbf{M})}^{\mathrm{I}}\right)} \\
& =P_{k} \\
& \quad \cdot\left(1-f\left(\rho, \mathrm{I}^{\Theta}\right)+f\left(\rho, \mathrm{I}^{\Theta}\right) \cdot\left(1-f\left(\rho, \mathrm{II}^{\Theta}\right)\right)\right) \\
& =1-f\left(\rho, \mathrm{I}^{\Theta}\right) f\left(\rho, \mathrm{II}^{\Theta}\right) .
\end{aligned}
$$

Since the expansion of $e^{x}$ is $1+x+x^{2} / 2 !+\cdots+x^{n} / n !$, then the approximate representations of $f(\rho, \mathrm{I}), f(\rho, \mathrm{II}), f\left(\rho, \mathrm{I}^{\Theta}\right)$, and $f\left(\rho, \mathrm{II}^{\Theta}\right)$ are

$$
\begin{aligned}
f(\rho, \mathrm{I}) & \approx\left(\frac{\rho_{(\mathrm{I})}^{n}}{n !}\right)\left(e^{\rho_{(\mathrm{I})}}\right)^{-1}, \\
f(\rho, \mathrm{II}) & \approx\left(\frac{\rho_{\mathrm{II}}^{n_{i+1}}}{n !}\right)\left(e^{\rho_{\mathrm{II}}}\right)^{-1}, \\
f\left(\rho, \mathrm{I}^{\Theta}\right) & \approx\left(\frac{\rho_{\left(\mathrm{I}^{\Theta}\right)}^{n}}{n !}\right)\left(e^{\rho_{\left(\mathrm{I}^{\Theta}\right)}}\right)^{-1}, \\
f\left(\rho, \mathrm{II}^{\Theta}\right) & =\left(\frac{\left.\rho_{(\mathrm{II}}^{n}\right)}{n !}\right)\left(e^{\left.\rho_{(\mathrm{II}}^{\Theta}\right)}\right)^{-1} .
\end{aligned}
$$

In order to prove Theorem 4 , we need to prove

$$
\begin{aligned}
& 1-f(\rho, \mathrm{I}) f(\rho, \mathrm{II}) \geq 1-f\left(\rho, \mathrm{I}^{\Theta}\right) f\left(\rho, \mathrm{II}^{\Theta}\right) \Longleftarrow f\left(\rho, \mathrm{I}^{\Theta}\right) f\left(\rho, \mathrm{II}^{\Theta}\right)-f(\rho, \mathrm{I}) f(\rho, \mathrm{II}) \geq 0 \Longleftarrow \frac{f\left(\rho, \mathrm{I}^{\Theta}\right) f\left(\rho, \mathrm{II}^{\Theta}\right)}{f(\rho, \mathrm{I}) f(\rho, \mathrm{II})} \\
& \geq 1 \text {, } \\
& \left.\frac{f\left(\rho, \mathrm{I}^{\Theta}\right) f\left(\rho, \mathrm{II}^{\Theta}\right)}{f(\rho, \mathrm{I}) f(\rho, \mathrm{II})}=\frac{\left(\left(\rho_{\left(\mathrm{I}^{\Theta}\right)}^{n} / n !\right)\left(e^{\rho_{\left(\mathrm{I}^{\Theta}\right)}}\right)^{-1}\right)\left(\left(\rho_{(\mathrm{II})}^{n} / n !\right)\left(e^{\left.\rho_{(\mathrm{II}}^{\Theta}\right)}\right)^{-1}\right)}{\left(\left(\rho_{(\mathrm{I})}^{n} / n !\right)\left(e^{\rho_{(\mathrm{I})}}\right)^{-1}\right)\left(\left(\rho_{\mathrm{II}}^{n_{i}} / n !\right)\left(e^{\rho_{(\mathrm{II})}}\right)^{-1}\right)}=\left(\frac{\left.\rho_{\left(\mathrm{I}^{\Theta}\right)}^{n} \rho_{(\mathrm{II}}^{n}\right)}{\rho_{(\mathrm{I})}^{n} \rho_{(\mathrm{II})}^{n}}\right) e^{\left(\rho_{(\mathrm{I})}+\rho_{(\mathrm{II})}-\rho_{\left(\mathrm{I}^{\Theta}\right)}-\rho_{(\mathrm{II}}^{\Theta}\right)}\right)
\end{aligned}
$$

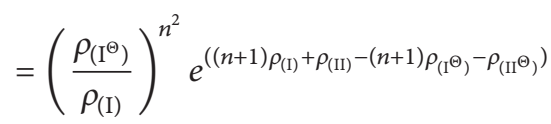

$$
\begin{aligned}
& =\left(\frac{\lambda t_{\text {smean }(\mathrm{II})} P_{f(\mathrm{II})}}{\lambda t_{s \operatorname{smean}(\mathrm{I})} P_{f(\mathrm{I})}}\right)^{n^{2}} e^{\lambda\left((n+1) t_{s m e a n(\mathrm{I})} P_{f(\mathrm{I})}+\left(1-E_{(\mathrm{I})}\right) t_{s \operatorname{mean}(\mathrm{II})} P_{f(\mathrm{II})}-(n+1) t_{\text {smean }(\mathrm{II})} P_{f(\mathrm{II})}-\left(1-E_{(\mathrm{II})}\right) t_{s \operatorname{mean}(\mathrm{I})} P_{f(\mathrm{I})}\right)}
\end{aligned}
$$

Let $t_{\text {smean(II) }} P_{f(\mathrm{II})}=A$ and $t_{\text {smean(I) }} P_{f(\mathrm{I})}=B$; then,

$$
\begin{aligned}
& \frac{f\left(\rho, \mathrm{I}^{\Theta}\right) f\left(\rho, \mathrm{II}^{\Theta}\right)}{f(\rho, \mathrm{I}) f(\rho, \mathrm{II})} \\
& \quad=\left(\frac{A}{B}\right)^{n^{2}} e^{\lambda\left((n+1) B+\left(1-E_{(\mathrm{I})}\right) A-(n+1) A-\left(1-E_{(\mathrm{II})}\right) B\right)} .
\end{aligned}
$$

Since $P_{f(\mathrm{II})}=P_{f(\mathrm{I})}$, then $A>B$, and the result of Theorem 4 follows.

The terms $A$ and $B$ that appear in the proof of Theorem 4 could be a factor in channels allocation; we extended Theorem 4 to obtain Lemma 5.

Lemma 5. Let $\mathbf{E}_{\left(\mathbf{M}, \mathbf{n}_{(M)}\right)}$ be the DE of the M-layer BMDS; $\mathbf{n}_{(\mathbf{M})}=\left(n_{1}, n_{2}, \ldots, n_{M}\right)$ is the allocation plan, $n_{i}$ is the number of target channels deployed along the ith layer, and let $n_{1}=n_{2}=$ $\cdots=n_{M}$. Allocation plan $\mathbf{n}_{(\mathbf{M})}^{I}=(I, I I)$ indicates that defense weapons of type I are forward-deployed, and $\mathbf{n}_{(\mathbf{M})}^{I I}=(I I, I)$ indicates that defense weapons of type II are forward-deployed. Suppose that defense weapons are identical in the same layer, and $P_{k(I)}=P_{k(I I)}$; if $P_{f(I)} t_{\text {smean (I) }} \leq P_{f(I I)} t_{\text {smean(II) }}$, then one has $\mathbf{E}_{\left(\mathbf{M}, \mathbf{n}_{(\mathbf{M})}^{I}\right)} \geq \mathbf{E}_{\left(\mathbf{M}, \mathbf{n}_{(\mathbf{M})}^{I I}\right)}$.

4.2.2. Different Defense Weapons and Different SSKPs. In this section, we consider different defense weapons and different SSKPs of layered BMDS. It is obvious that higher SSKP dose improves the DE. How SSKP affects the target channels allocation is a complex problem; however, we can summarize some useful rules; see Scenario 4.

Scenario 4. Suppose that the number of layers is 3 , the number of defense weapon types is 2 (types I and II), the probability that BMs will be detected by radars is $0.8, \lambda=$ $5 \mathrm{BMs} / \mathrm{min}, t_{s \text { mean }(\mathrm{I})}=t_{\text {smean }(\mathrm{II})}=0.75 \mathrm{~min}, P_{k(\mathrm{I})}=0.8$, and $P_{k(\mathrm{II})}=0.6$.

Table 7 is the DE of three-layer defense. It can be seen from Table 7 that plan $\mathbf{n}_{(3)}=$ (I, I, II) has the biggest DE. We also found that $\mathbf{n}_{(4)}=(\mathrm{I}, \mathrm{I}, \mathrm{I}, \mathrm{II})$ is the best, and then we have Theorem 6. 
TABLE 7: DE of three-layer defense.

\begin{tabular}{lccccrr}
\hline 1st-layer type & 1st-layer DE & 2nd-layer type & 2nd-layer DE & 3rd-layer type & 3rd-layer DE & Total DE \\
\hline II & $36.720 \%$ & II & $41.400 \%$ & I & 0.594 & $83.930 \%$ \\
II & $42.120 \%$ & I & $59.040 \%$ & II & 0.400 & $84.766 \%$ \\
II & $42.600 \%$ & I & $55.840 \%$ & I & 0.506 \\
I & $54.240 \%$ & II & $39.600 \%$ & II & 0.415 & $85.837 \%$ \\
I & $\mathbf{5 6 . 6 4 0 \%}$ & I & $\mathbf{5 4 . 4 0 0 \%}$ & II & $\mathbf{0 . 4 2 6}$ \\
\hline
\end{tabular}

Theorem 6. Let $\mathbf{E}_{\left(\mathrm{M}, \mathbf{n}_{(M)}\right)}$ be the DE of the M-layer BMDS; $\mathbf{n}_{(\mathbf{M})}=\left(n_{1}, n_{2}, \ldots, n_{M}\right)$ is the allocation plan, $n_{i}$ is the number of target channels deployed along the ith layer, and let $n_{1}=n_{2}=$ $\cdots=n_{M}$. Allocation plan $\mathbf{n}_{(\mathbf{M})}^{I}=(I, I I)$ indicates that defense weapons of type I are forward-deployed, and $\mathbf{n}_{(\mathrm{M})}^{I I}=(I I, I)$ indicates that defense weapons of type II are forward-deployed. Suppose that defense weapons are identical in the same layer, and $t_{\text {smean }(I)}=t_{\text {smean }(I))}$ and $P_{f(I)}=P_{f(I I)}$; if $P_{k(I)}>P_{k(I I)}$, then one has $\mathbf{E}_{\left(\mathbf{M}, \mathbf{n}_{(\mathbb{M})}^{I}\right)} \geq \mathbf{E}_{\left(\mathbf{M}, \mathbf{n}_{(\mathbb{M})}^{I I}\right)}$.

Proof. Suppose that we have an allocation plan $\mathbf{n}_{(\mathbf{M})}^{\mathrm{I}^{\Theta}}=$ $\left(\mathrm{I}^{\Theta}, \mathrm{II}^{\Theta}\right)$, where $P_{\mathrm{k}\left(\mathrm{I}^{\Theta}\right)}=P_{k(\mathrm{II})}$ and $P_{k(\mathrm{II})}{ }^{\Theta}=P_{k(\mathrm{I})}$. In order to prove Theorem 4, we only need to prove $\mathbf{E}_{\left(\mathbf{M}, \mathbf{n}_{(M)}^{\mathrm{I}}\right)} \geq \mathbf{E}_{\left(\mathbf{M}, \mathbf{n}_{(\mathbf{M})}^{\mathrm{\Theta}}\right)}$.

$f(\rho, \mathrm{I}), f(\rho, \mathrm{II}), f\left(\rho, \mathrm{I}^{\Theta}\right)$, and $f\left(\rho, \mathrm{II}^{\Theta}\right)$ are

$$
\begin{gathered}
f(\rho, \mathrm{I})=\left(\frac{\rho_{(\mathrm{I})}^{n}}{n !}\right)\left(\sum_{k=0}^{n} \frac{\rho_{(\mathrm{I})}^{k}}{k !}\right)^{-1}, \\
f(\rho, \mathrm{II})=\left(\frac{\rho_{(\mathrm{II})}^{n}}{n !}\right)\left(\sum_{k=0}^{n} \frac{\rho_{(\mathrm{II})}^{k}}{k !}\right)^{-1}, \\
f\left(\rho, \mathrm{I}^{\Theta}\right)=\left(\frac{\rho_{\left(\mathrm{I}^{\Theta}\right)}^{n}}{n !}\right)\left(\sum_{k=0}^{n} \frac{\rho_{\left(\mathrm{I}^{\Theta}\right)}^{k}}{k !}\right)^{-1}, \\
f\left(\rho, \mathrm{II}^{\Theta}\right)=\left(\frac{\left.\rho_{(\mathrm{II})}^{n}\right)}{n !}\right)\left(\sum_{k=0}^{n} \frac{\rho_{\left(\mathrm{II}^{\Theta}\right)}^{k}}{k !}\right)^{-1},
\end{gathered}
$$

where

$$
\begin{aligned}
\rho_{(\mathrm{I})} & =\lambda \cdot t_{s m e a n(\mathrm{I})} \cdot P_{f(\mathrm{I})}, \\
\rho_{\left(\mathrm{I}^{\Theta}\right)} & =\lambda \cdot t_{s \operatorname{smean}\left(\mathrm{I}^{\Theta}\right)} \cdot P_{f\left(\mathrm{I}^{\Theta}\right)}, \\
\rho_{(\mathrm{II})} & =\left(1-E_{(\mathrm{I})}\right) \lambda t_{s \operatorname{mean}(\mathrm{II})} P_{f(\mathrm{II})}, \\
\rho_{(\mathrm{II})} & =\left(1-E_{\left(\mathrm{I}^{\Theta}\right)}\right) \lambda t_{s \operatorname{mean}(\mathrm{II})} P_{f\left(\mathrm{(}^{\Theta}\right)}, \\
E_{(\mathrm{I})} & =(1-f(\rho, \mathrm{I})) \cdot P_{k(\mathrm{I})}, \\
E_{\left(\mathrm{I}^{\Theta}\right)} & =\left(1-f\left(\rho, \mathrm{I}^{\Theta}\right)\right) \cdot P_{k(\mathrm{II})} .
\end{aligned}
$$

$\mathrm{DE}$ of the defense are

$$
\begin{aligned}
E_{\left(M, n_{(M)}^{\mathrm{I}}\right)}= & P_{k(\mathrm{I})}-f(\rho, \mathrm{I}) P_{k(\mathrm{I})}+P_{k(\mathrm{II})} \\
& -f(\rho, \mathrm{II}) P_{k(\mathrm{II})}-P_{k(\mathrm{I})} P_{k(\mathrm{II})} \\
& +f(\rho, \mathrm{II}) P_{k(\mathrm{I})} P_{k(\mathrm{II})} \\
& +f(\rho, \mathrm{I}) P_{k(\mathrm{I})} P_{k(\mathrm{II})} \\
& -f(\rho, \mathrm{I}) f(\rho, \mathrm{II}) P_{k(\mathrm{I})} P_{k(\mathrm{II})}, \\
E_{\left(M, n_{(M)}^{1^{\Theta}}\right)}= & \left.P_{k\left(\mathrm{I}^{\Theta}\right)}-f\left(\rho, \mathrm{I}^{\Theta}\right) P_{k\left(\mathrm{I}^{\Theta}\right)}+P_{k(\mathrm{II}}{ }^{\Theta}\right) \\
& -f\left(\rho, \mathrm{II}^{\Theta}\right) P_{k\left(\mathrm{I} \mathrm{I}^{\Theta}\right)}-P_{k\left(\mathrm{I}^{\Theta}\right)} P_{k\left(\mathrm{II}^{\Theta}\right)} \\
& +f\left(\rho, \mathrm{II}^{\Theta}\right) P_{k\left(\mathrm{I}^{\Theta}\right)} P_{k\left(\mathrm{II}^{\Theta}\right)} \\
& \left.+f\left(\rho, \mathrm{I}^{\Theta}\right) P_{k\left(\mathrm{I}^{\Theta}\right)} P_{k(\mathrm{II}}{ }^{\Theta}\right) \\
& \left.-f\left(\rho, \mathrm{I}^{\Theta}\right) f\left(\rho, \mathrm{II}^{\Theta}\right) P_{k\left(\mathrm{I}^{\Theta}\right)} P_{k(\mathrm{II}}\right) .
\end{aligned}
$$

In order to prove Theorem 6, we need to prove

$$
\begin{gathered}
E_{\left(M, n_{(M)}^{\mathrm{I}}\right)}-E_{\left(M, n_{(M)}^{\mathrm{\Theta}}\right)}=P_{k(\mathrm{I})} P_{k(\mathrm{II})}(f(\rho, \mathrm{II})+f(\rho, \mathrm{I}) \\
-f(\rho, \mathrm{I}) f(\rho, \mathrm{II})-f\left(\rho, \mathrm{II}^{\Theta}\right)-f\left(\rho, \mathrm{I}^{\Theta}\right) \\
\left.+f\left(\rho, \mathrm{I}^{\Theta}\right) f\left(\rho, \mathrm{II}^{\Theta}\right)\right)-f(\rho, \mathrm{I}) P_{k(\mathrm{I})}-f(\rho, \mathrm{II}) \\
\cdot P_{k(\mathrm{II})}+f\left(\rho, \mathrm{I}^{\Theta}\right) P_{k(\mathrm{II})}+f\left(\rho, \mathrm{II}^{\Theta}\right) P_{k(\mathrm{I})} \geq 0 .
\end{gathered}
$$

Since $P_{k(\mathrm{I})}>P_{k(\mathrm{II})}, f(\rho, \mathrm{I})=f\left(\rho, \mathrm{I}^{\Theta}\right)$, then

$$
\begin{aligned}
& f(\rho, \mathrm{I})=f\left(\rho, \mathrm{I}^{\Theta}\right) \geq f\left(\rho, \mathrm{II}^{\Theta}\right) \geq f(\rho, \mathrm{II}), \\
& P_{k(\mathrm{I})} P_{k(\mathrm{II})}(1-f(\rho, \mathrm{I}))\left(f\left(\rho, \mathrm{II}^{\Theta}\right)-f(\rho, \mathrm{II})\right) \\
& \quad+f(\rho, \mathrm{I})\left(P_{k(\mathrm{I})}-P_{k(\mathrm{II})}\right)+f(\rho, \mathrm{II}) P_{k(\mathrm{II})} \\
& -f\left(\rho, \mathrm{II}^{\Theta}\right) P_{k(\mathrm{I})} \geq 0 .
\end{aligned}
$$




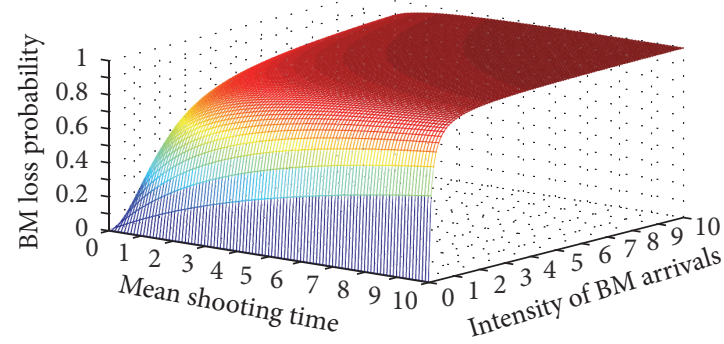

(a)

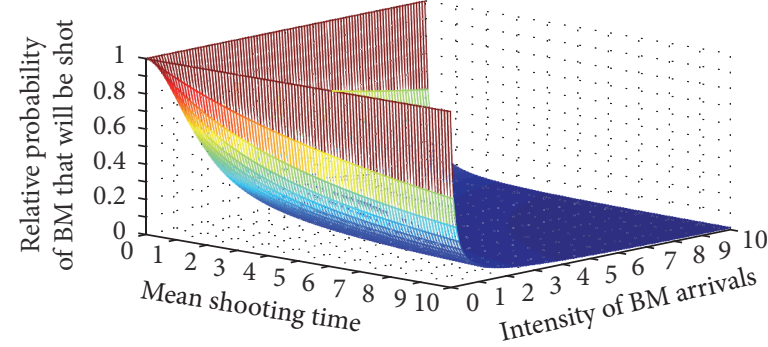

(b)

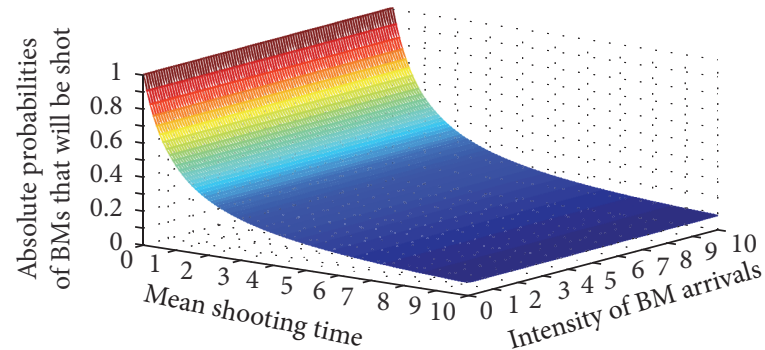

(c)

FIGURE 5: Performance parameters as functions of the mean shooting time and the intensity of BM arrivals (M/M/1/1 system).

Since the expansion of $e^{x}$ is $1+x+x^{2} / 2 !+\cdots+x^{n} / n !$, then the approximate representations of $f\left(\rho, \mathrm{I}^{\Theta}\right)$ and $f\left(\rho, \mathrm{II}^{\Theta}\right)$ are

$$
\begin{aligned}
& f\left(\rho, \mathrm{I}^{\Theta}\right) \approx\left(\frac{\rho_{\left(\mathrm{I}^{\Theta}\right)}^{n}}{n !}\right)\left(e^{\rho_{\left(\mathrm{I}^{\Theta}\right)}}\right)^{-1}, \\
& f\left(\rho, \mathrm{II}^{\Theta}\right)=\left(\frac{\rho_{(\mathrm{II})}^{n}}{n !}\right)\left(e^{\left.\rho_{(\mathrm{II}} \Theta\right)}\right)^{-1}, \\
& \frac{f\left(\rho, \mathrm{II}^{\Theta}\right)}{f\left(\rho, \mathrm{I}^{\Theta}\right)}=\left(1-\left(1-f\left(\rho, \mathrm{I}^{\Theta}\right)\right) \cdot P_{k\left(\mathrm{I}^{\Theta}\right)}\right)^{n} e^{\left.\rho_{\left(\mathrm{I}^{\Theta}\right)} \cdot \rho_{(\mathrm{II}}{ }^{\Theta}\right)} .
\end{aligned}
$$

Let $t_{\text {smean(II) }} P_{f(\mathrm{II})}=A$ and $t_{\text {smean }(\mathrm{I})} P_{f(\mathrm{I})}=B$; then,

$$
\begin{aligned}
& \frac{f\left(\rho, \mathrm{I}^{\Theta}\right) f\left(\rho, \mathrm{II}^{\Theta}\right)}{f(\rho, \mathrm{I}) f(\rho, \mathrm{II})} \\
& \quad=\left(\frac{A}{B}\right)^{n^{2}} e^{\lambda\left((n+1) B+\left(1-E_{(\mathrm{I})}\right) A-(n+1) A-\left(1-E_{(\mathrm{II})}\right) B\right)} .
\end{aligned}
$$

Obviously, we have

$$
\left(1-\left(1-f\left(\rho, \mathrm{I}^{\Theta}\right)\right) \cdot P_{k\left(\mathrm{I}^{\Theta}\right)}\right)^{n} e^{\left.\rho_{\left(\mathrm{I}^{\Theta}\right)} \cdot \rho_{(\mathrm{II}}^{\Theta}\right)} \longrightarrow 0 .
$$

The result of Theorem 4 follows.

\section{Numerical Examples}

In this section, we use numerical examples to generate some insights into the performance of the proposed queuing models in Section 3. Firstly, using the formula in Section 3.1.2, we draw the relationship of the mean shooting time and intensity of BM arrivals (see Figure 5). Then, we consider using the formula in Section 3.1.1 and calculate the loss probability of the $\mathrm{M} / \mathrm{M} / \mathrm{N} / \mathrm{N}$ system. We draw the relationship of the BM loss probability and offered density of shootings with $N=$ $1,2,3,4,5,6,8,12,16,20,30,40$, and 50 (see Figure 6). From Figures 5 and 6 , we can see that the probability of BM loss increases with the increasing mean shooting time and intensity of BM arrivals.

We set two scenarios: scenario 1 (the number of total BMs is 30 , the number of target channels is 3, SSKP is 0.7 , the probability that $\mathrm{BMs}$ will be detected by radars is 0.8 , $\lambda=3 \mathrm{BMs} / \mathrm{min}$, and $\left.t_{\text {smean }}=1 \mathrm{~min}(\mu=1)\right)$ and scenario 2 (the number of total BMs is 50 , the number of target channels is 8 , SSKP is 0.8 , the probability that BMs will be detected by radars is $0.9, \lambda=5 \mathrm{BMs} / \mathrm{min}$, and $\left.t_{\text {smean }}=1 \mathrm{~min}(\mu=1)\right)$. We will firstly simulate the $\mathrm{M} / \mathrm{M} / \mathrm{N} / \mathrm{N}$ queueing system and use Matlab to get the figure of the performance of the two scenarios (see Figure 7). Then, we will secondly simulate the $\mathrm{M} / \mathrm{M} / \mathrm{N} / \mathrm{C}$ queueing system and use Matlab to get the figure of the performance of the two scenarios (see Figure 8). Table 8 shows the results of operating parameters of the two queueing systems.

In order to explore the changes in the relationship between the performance of systems and different factors, we adjust the parameters in scenario 1 , that is, (a) increase the number of arriving BMs, (b) increase the intensity of arriving BMs, (c) increase the mean shooting time for each BM, and (d) reduce the number of target channels. Figure 9 is the queue length as functions of the number of BMs, and Table 9 shows the results of operating parameters of the adjusted queueing system.

Through adjustment of the system configuration, we can observe and summarize the queueing system running condition. This can be useful for decision-making of BMDS operation control and adjusting system configuration. 


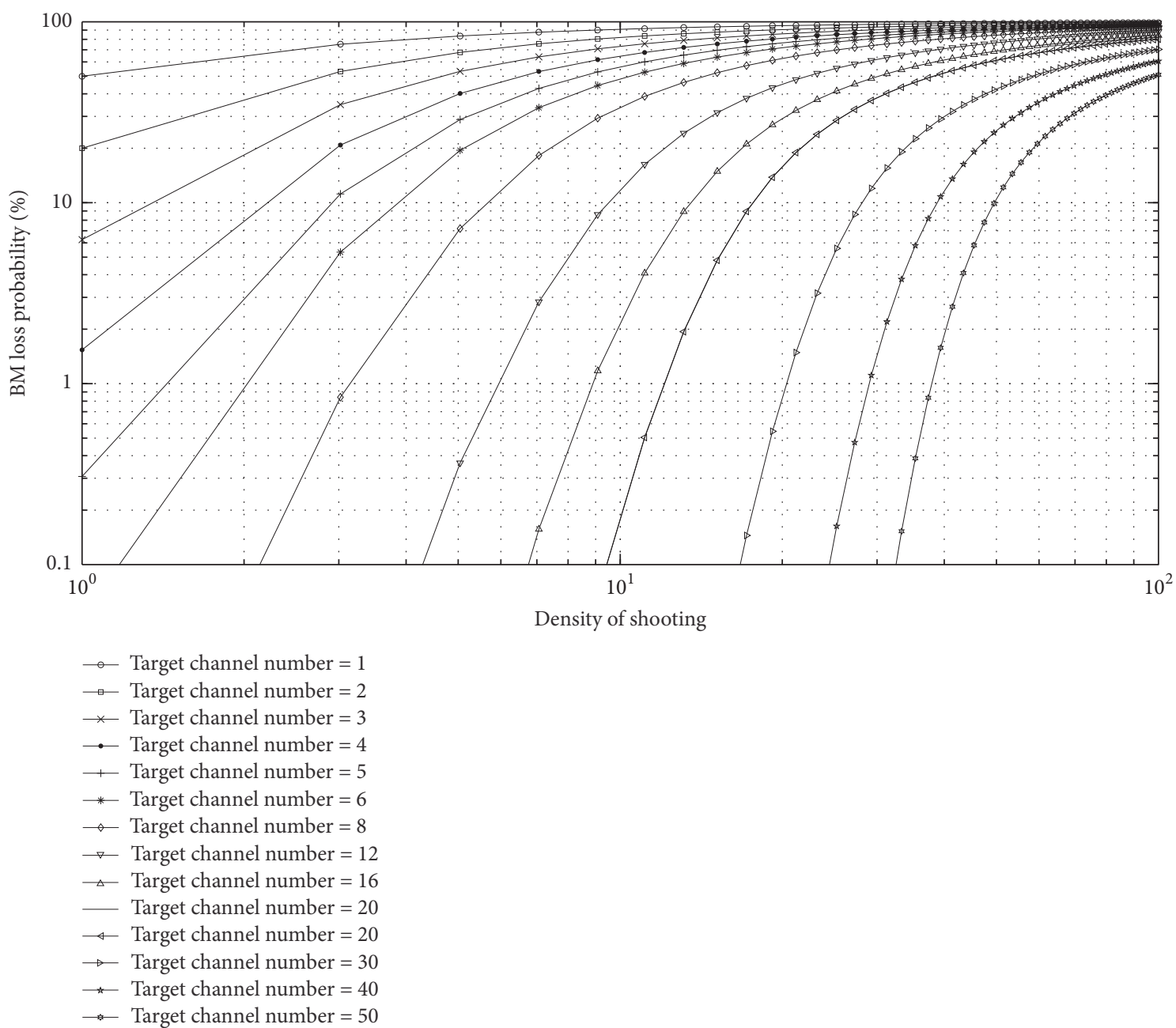

FIgURE 6: The probability of BM loss as functions of the number of target channels and density of shooting.

TABLE 8: Results of operating parameters of the two queueing systems.

\begin{tabular}{|c|c|c|c|c|}
\hline \multirow{2}{*}{ Parameters } & \multicolumn{2}{|c|}{$\mathrm{M} / \mathrm{M} / \mathrm{N} / \mathrm{N}$} & \multicolumn{2}{|c|}{$\mathrm{M} / \mathrm{M} / \mathrm{N} / \mathrm{C}$} \\
\hline & Scenario 1 & Scenario 2 & Scenario 1 & Scenario 2 \\
\hline Mean occupancy rate of target channels & $22.6 \%$ & $10.6 \%$ & $64.5 \%$ & $1.7 \%$ \\
\hline Mean number of waiting BMs & 0.000 & 0.000 & 1.847 & 0.000 \\
\hline Mean waiting time of BMs & 0.000 & 0.000 & 0.801 & 0.000 \\
\hline Mean number of BMs in system & 1.732 & 5.018 & 4.326 & 3.830 \\
\hline Number of penetrated BMs & 7 & 3 & 0 & 0 \\
\hline Probability of BM penetration & $23.3 \%$ & $6.0 \%$ & $0 \%$ & $0 \%$ \\
\hline Total DE & $42.95 \%$ & $67.68 \%$ & $56 \%$ & $72 \%$ \\
\hline
\end{tabular}

\section{Concluding Remarks and Future Work}

In this paper, we presented a theory for the representation, modeling, and performance simulation of the layered BMDS $\mathrm{M} / \mathrm{M} / \mathrm{N}$ queueing systems. In addition to the queueing model, four simple rules have been developed for use on the complex channel allocation problems. The main aim of this work is to study a stochastic missile defense process close to the Poisson process and to find allocation rules that maximize DE or minimize the cost to achieve a required DE. In Section 4.1.2, we also proposed a novel and robust search algorithm for obtaining the minimum requirements across a set of neighborhoods. Numerical examples using models in Section 2 revealed some guidelines for adjusting queueing parameters.

This work may help to understand the rules of queueing process and to provide optimal configuration suggestions for defense decision-making. The work can easily be expanded 


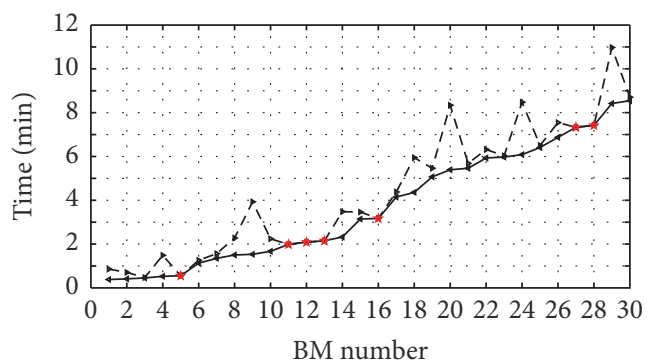

$\leftarrow$ Arrival time of $\mathrm{BM}$

$\rightarrow-$ Finish time of shooting

* BM penetration

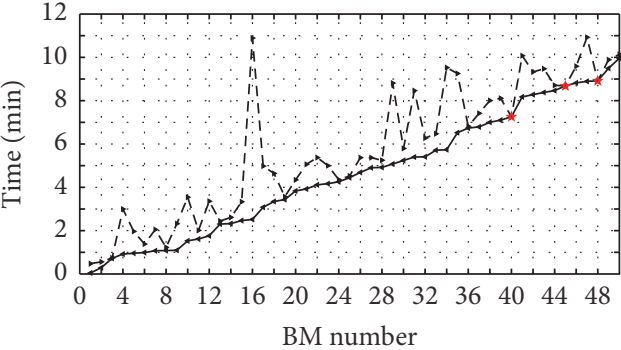

—. Arrival time of BM

$\rightarrow-$ Finish time of shooting

* BM penetration

(a) BM arrival and finish time of scenario 1 and scenario 2
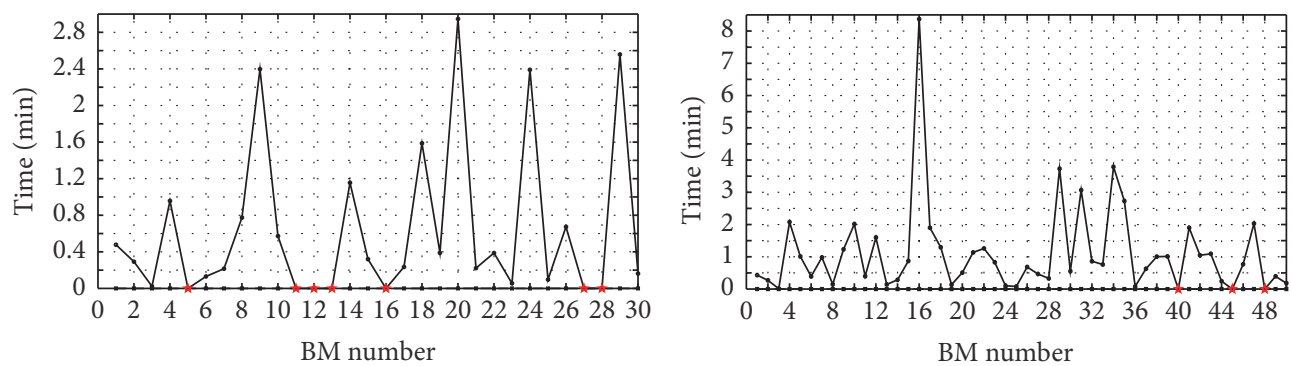

$\ldots$ Waiting time
$\ldots$ Shooting time
$\star \quad$ BM penetration

-. - Waiting time

$\rightarrow$ Shooting time

* BM penetration

(b) BM waiting time and weapons shooting time of scenario 1 and scenario 2
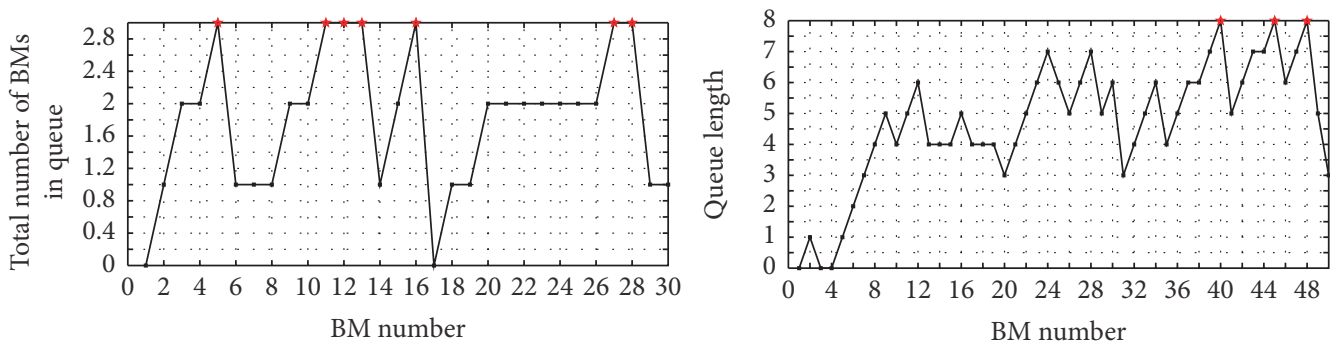

- Total number of BMs in queue

* BM penetration

- Total number of BMs in queue

* BM penetration

(c) Queue length of scenario 1 and scenario 2

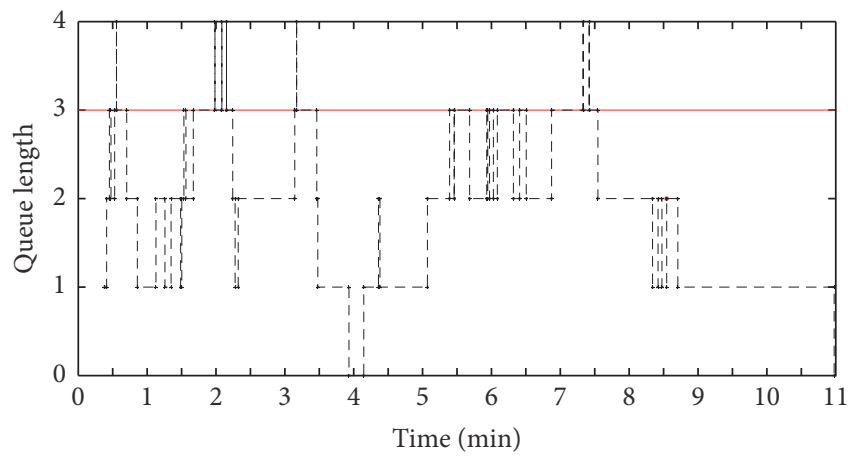

- Last BM arrived

— Maximum capacity of system

-.. Total number of BMs in queue

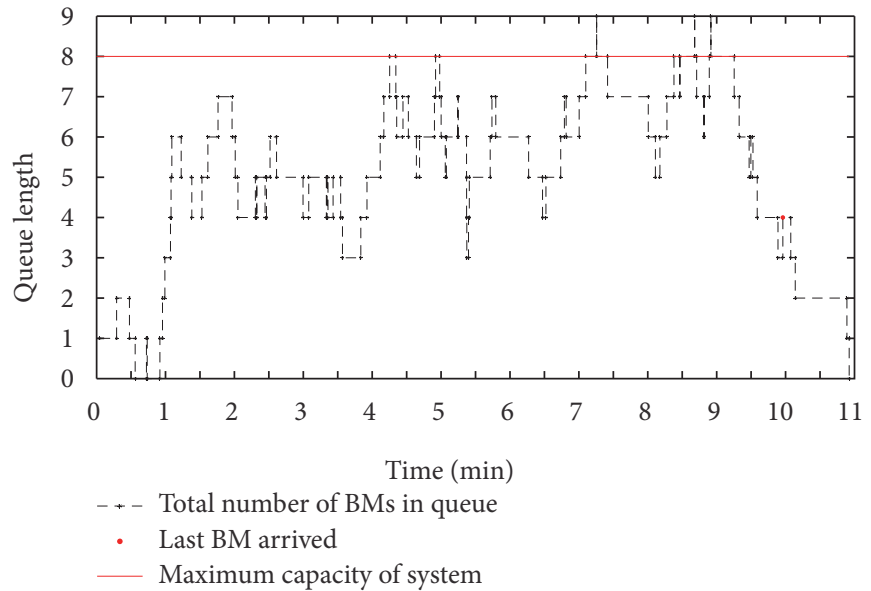

(d) Total number of BMs in queue and last BM that arrived of scenario 1 and scenario 2

FIGURE 7: Figures of the performance of the M/M/N/N queueing system. 

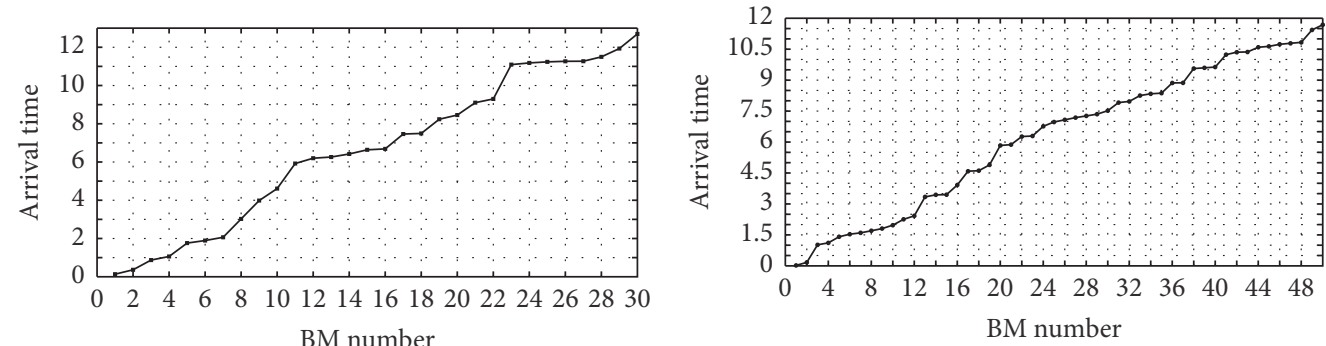

(a) BM arrival time of scenario 1 and scenario 2
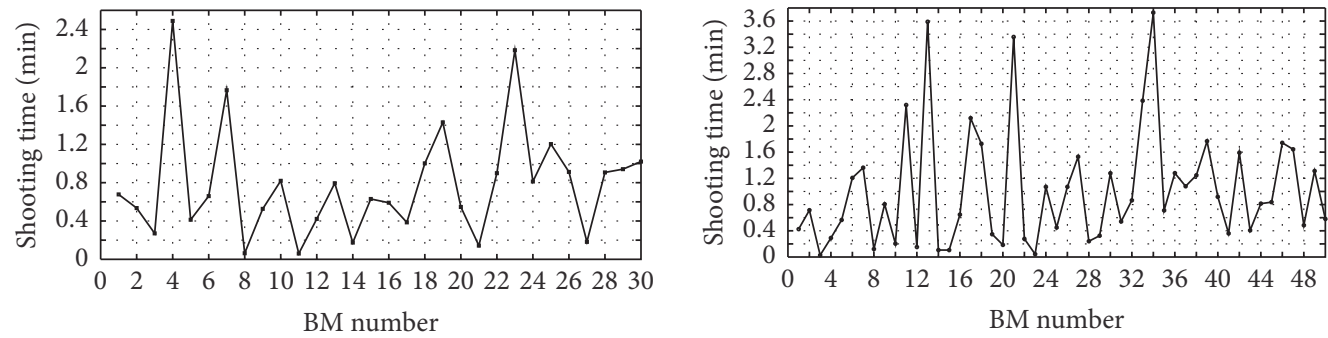

(b) Shooting time for each $\mathrm{BM}$ of scenario 1 and scenario 2
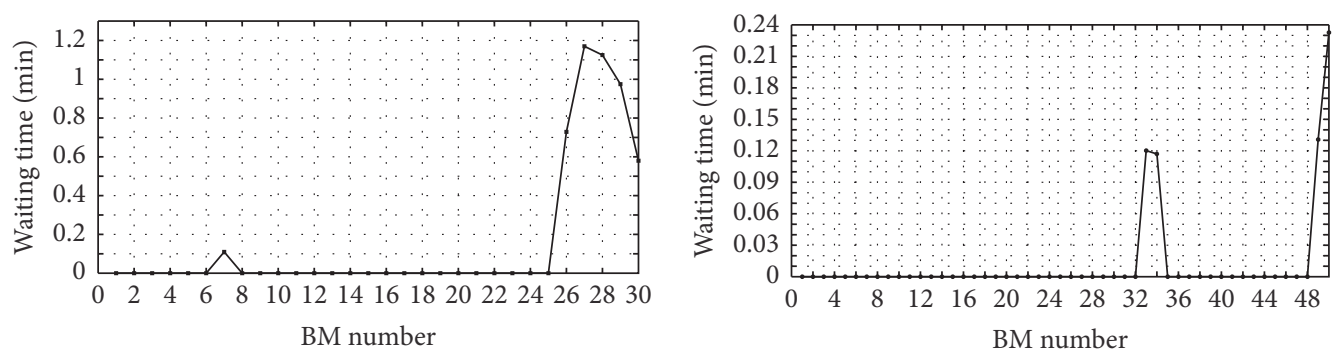

(c) Waiting time of each $\mathrm{BM}$ of scenario 1 and scenario 2
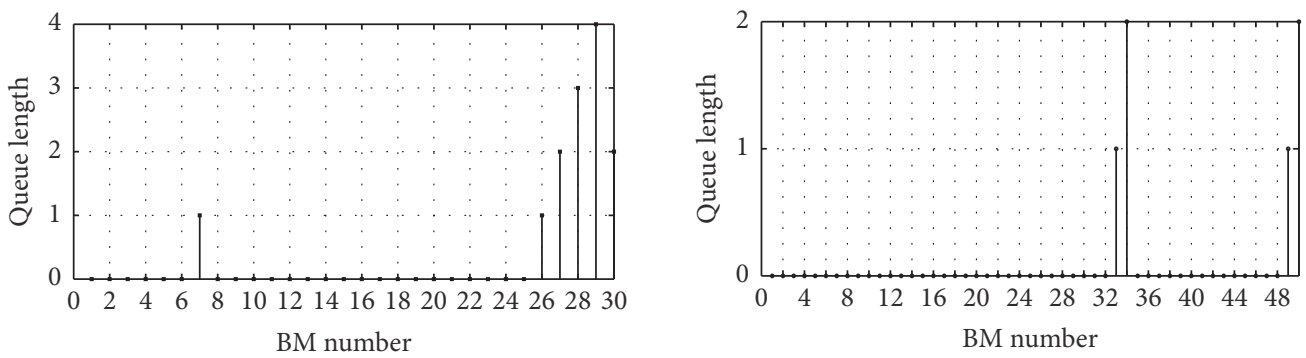

(d) Queue length of scenario 1 and scenario 2
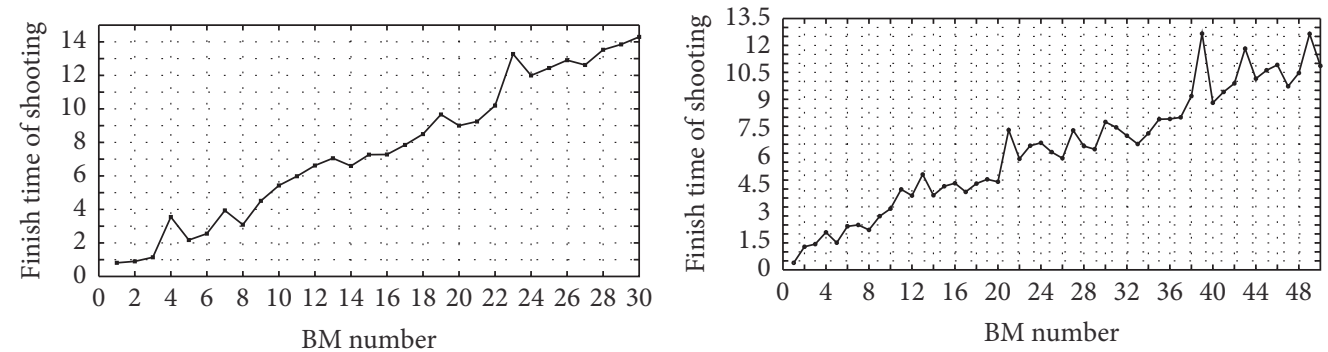

(e) Finish time of shooting of scenario 1 and scenario 2

FIGURE 8: Figures of the performance of the M/M/N/C queueing system. 


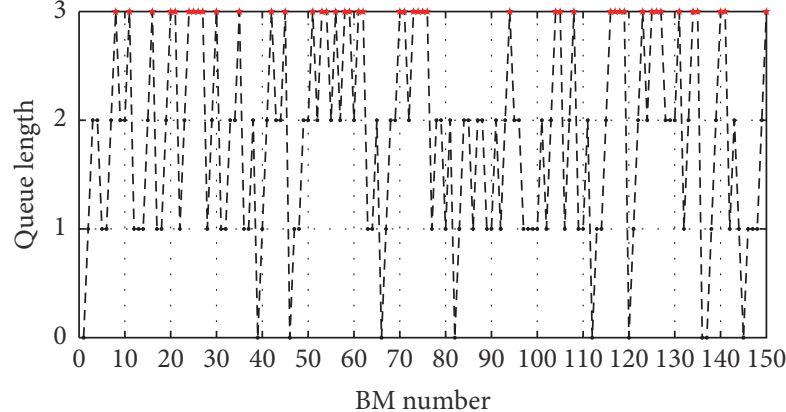

-.- Total number of BMs in system

* BM penetration

(a) 3-fold increase in the number of arriving BMs

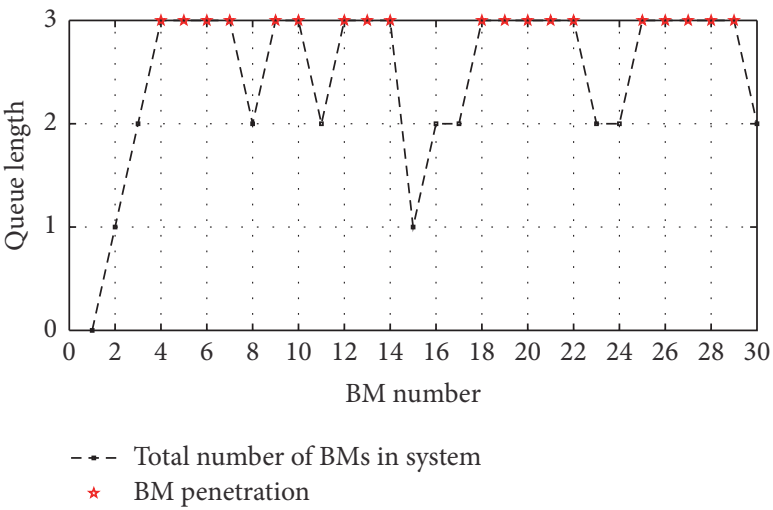

(c) 2-fold increase in the mean shooting time for each BM

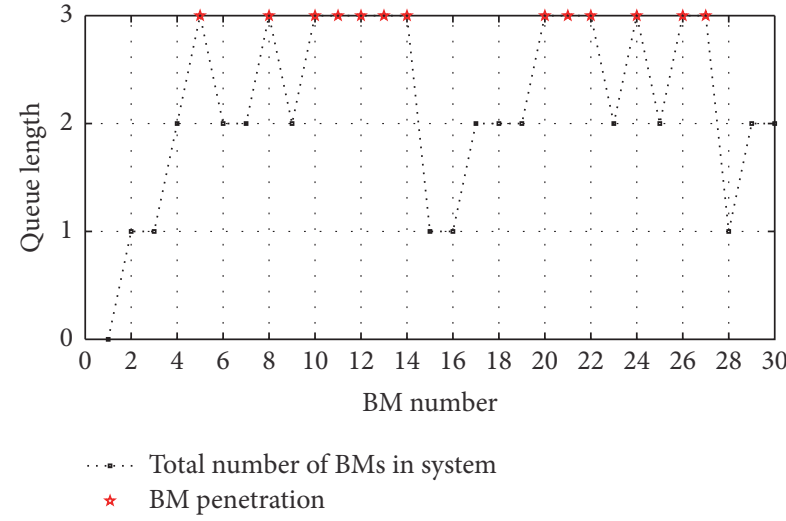

(b) 2-fold increase in the intensity of arriving BMs

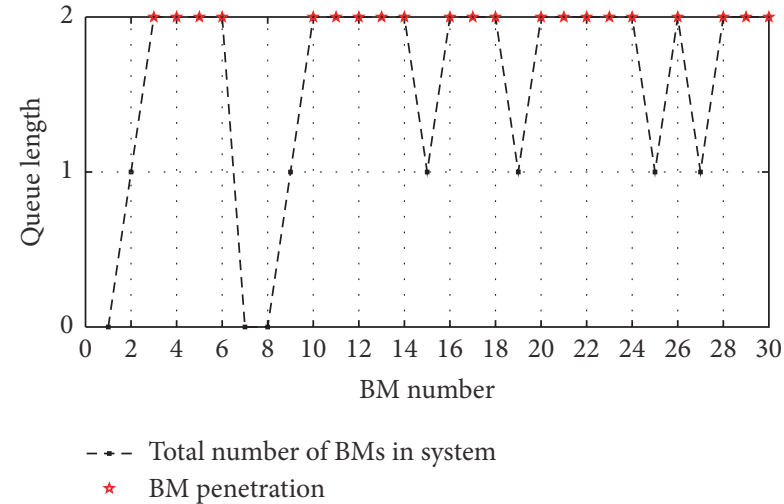

(d) $1 / 3$ reduction in the number of target channels

Figure 9: The queue length as functions of the number of BMs.

TABLE 9: The results of operating parameters of the adjusted queueing system.

\begin{tabular}{|c|c|c|c|c|c|}
\hline Parameters & Before adjustment & $\begin{array}{l}\text { Number of BMs, } \\
\text { 3-fold increase }\end{array}$ & $\begin{array}{l}\text { Intensity of BMs } \\
\text { arriving, 2-fold increase }\end{array}$ & $\begin{array}{l}\text { Mean shooting time, } \\
\text { 2-fold increase }\end{array}$ & $\begin{array}{c}\text { Target } \\
\text { channels, } 1 / 3 \\
\text { reduction }\end{array}$ \\
\hline $\begin{array}{l}\text { Mean occupancy rate of } \\
\text { target channels }\end{array}$ & $22.6 \%$ & $27.7 \%$ & $40.9 \%$ & $43.9 \%$ & $63.9 \%$ \\
\hline $\begin{array}{l}\text { Mean number of waiting } \\
\text { BMs }\end{array}$ & 0.000 & 0.000 & 0.000 & 0.000 & 0.000 \\
\hline Mean waiting time of BMs & 0.000 & 0.000 & 0.000 & 0.000 & 0.000 \\
\hline $\begin{array}{l}\text { Mean number of BMs in } \\
\text { system }\end{array}$ & 1.732 & 1.887 & 2.059 & 2.084 & 1.491 \\
\hline Number of penetrated BMs & 7 & 45 & 15 & 19 & 21 \\
\hline $\begin{array}{l}\text { Probability of BM } \\
\text { penetration }\end{array}$ & $23.3 \%$ & $\begin{array}{c}30 \% \\
28.7 \% \text { increase }\end{array}$ & $\begin{array}{c}50 \% \\
114 \% \text { increase }\end{array}$ & $\begin{array}{c}63.3 \% \\
171 \% \text { increase }\end{array}$ & $\begin{array}{c}70 \% \\
200 \% \text { increase }\end{array}$ \\
\hline Total DE & $42.95 \%$ & $\begin{array}{c}39.2 \% \\
8.7 \% \text { reduction }\end{array}$ & $\begin{array}{c}28 \% \\
34.8 \% \text { reduction }\end{array}$ & $\begin{array}{c}20.6 \% \\
50.0 \% \text { reduction }\end{array}$ & $\begin{array}{c}16.8 \% \\
60.9 \% \\
\text { reduction }\end{array}$ \\
\hline
\end{tabular}

and continued to handle a broader array of queueing scenarios. Several areas for potentially valuable future research have emerged from this work; we suggest the following areas of further research [22-26]. (1) We proposed some approximation in our computations; an important question is the discussion of the accuracy of BM arrivals distribution.
The Poisson arrival process is not the only fitting model for the provided queueing model in our paper. We will consider Bernoulli or Markov BM arrival process in our future research. This also includes relaxing the assumptions of exponential shooting times and allowing waiting times to vary by BM and defense weapon. (2) For the convenience of 
calculation and verification, the 2 scenarios in Section 5 are simple and need further analysis in the follow-up work. (3) One such suggestion is an exploration into expanding the allocation rules presented in Section 4. (4) When the size of (41)-(56) is very large, an approximate solution can be obtained by using some advanced algorithms, for example, genetic, neural network, and heuristic algorithms.

\section{Competing Interests}

The authors declare that they have no competing interests.

\section{References}

[1] L. Li, F. Liu, G. Long, P. Guo, and X. Bie, "Modified particle swarm optimization for BMDS interceptor resource planning," Applied Intelligence, vol. 44, no. 3, pp. 471-488, 2016.

[2] L. Y. Li, F. X. Liu, G. Z. Long, P. Guo, and Y. Mei, "Intercepts allocation for layered defense," Journal of Systems Engineering and Electronics, vol. 27, no. 3, pp. 602-611, 2016.

[3] L. Evers, A. I. Barros, and H. Monsuur, "The cooperative ballistic missile defence game," in Decision and Game Theory for Security: 4th International Conference, GameSec 2013, Fort Worth, TX, USA, November 11-12, 2013. Proceedings, vol. 8252 of Lecture Notes in Computer Science, pp. 99-118, Springer, Berlin, Germany, 2013.

[4] G. Brown, M. Carlyle, D. Diehl, J. Kline, and K. Wood, "A two-sided optimization for theater ballistic missile defense," Operations Research, vol. 53, no. 5, pp. 745-763, 2005.

[5] L.-Y. Li, F.-X. Liu, and L.-F. Zhao, "Direct interceptor allocation method in antimissile firepower planning for multiple wave targets," Systems Engineering and Electronics, vol. 36, no. 11, pp. 2206-2212, 2014.

[6] W. R. Nunn, D. V. Glass, I. Hsu, and D. A. Perin, "Technical Note-Analysis of a Layered Defense Model," Operations Research, vol. 30, no. 3, pp. 595-599, 1982.

[7] S. Park and L. Rothrock, "Systematic analysis of framing bias in missile defense: implications toward visualization design," European Journal of Operational Research, vol. 182, no. 3, pp. 1383-1398, 2007.

[8] D. K. Al-Mutairi, A. K. Nanda, and R. M. Soland, "Monotonicity and comparability in ballistic missile defense systems," Journal of Statistical Planning and Inference, vol. 141, no. 7, pp. 2191-2200, 2011.

[9] J.-Y. Menq, P.-C. Tuan, and T.-S. Liu, "Discrete Markov ballistic missile defense system modeling," European Journal of Operational Research, vol. 178, no. 2, pp. 560-578, 2007.

[10] A. K. Erlang, "The theory of probabilities and telephone conversations," Nyt Tidsskrift for Matematik B, vol. 20, pp. 3339, 1909.

[11] R. Yang, S. Bhulai, and R. van der Mei, "Structural properties of the optimal resource allocation policy for single-queue systems," Annals of Operations Research, vol. 202, no. 1, pp. 211233, 2013.

[12] R. Kumar, M. E. Lewis, and H. Topaloglu, "Dynamic service rate control for a single-server queue with Markov-modulated arrivals," Naval Research Logistics, vol. 60, no. 8, pp. 661-677, 2013.

[13] I.-H. Hou and P. R. Kumar, "Queueing systems with hard delay constraints: a framework for real-time communication over unreliable wireless channels," Queueing Systems, vol. 71, no. 12, pp. 151-177, 2012.

[14] N. S. Shcherbakov, "Management decision-making algorithm for the ship protection against air attacks under risk conditions," Electromagnetic Waves and Electronic Systems, vol. 3, pp. 19-21, 2012.

[15] S.-S. Pan, D.-W. Ma, and Z.-G. Li, "Queueing network method for the combat effectiveness analysis of mixed-mode air-defense systems," Acta Armamentarii, vol. 27, no. 5, pp. 862-864, 2006.

[16] F.-X. Lu, L. Wu, and H.-Y. Wang, "Calculation of penetration probability of a target for a multi-line defense system," Systems Engineering and Electronics, vol. 27, no. 6, pp. 1061-1065, 2005.

[17] R. Hassin, Y. Y. Shaki, and U. Yovel, "Optimal service-capacity allocation in a loss system," Naval Research Logistics, vol. 62, no. 2, pp. 81-97, 2015.

[18] L. Lipsky, Queueing Theory-A Linear Algebraic Approach, Springer Science \& Business Media, New York, NY, USA, 2nd edition, 2008.

[19] L. Lakatos, L. Szeidl, and M. Telek, Introduction to Queueing Systems with Telecommunication Applications, Springer Science+Business Media, New York, NY, USA, 2012.

[20] S. S. Chen and L. Zhang, "Analysis on multiplayer defense of the different types of air-defense Weapons' disposition," Information Command Control System \& Simulation Technology, vol. 27, no. 4, pp. 49-52, 2007.

[21] S. S. Chen, Analysis and research on battlefield situation of air-defense operation [Ph.D. thesis], Air Force Engineering University, 2005.

[22] J. Anselmi and G. Casale, "Heavy-traffic revenue maximization in parallel multiclass queues," Performance Evaluation, vol. 70, no. 10, pp. 806-821, 2013.

[23] G. Guadagni, S. Ndreca, and B. Scoppola, "Queueing systems with pre-scheduled random arrivals," Mathematical Methods of Operations Research, vol. 73, no. 1, pp. 1-18, 2011.

[24] Z. Liu, L. Wynter, C. H. Xia, and F. Zhang, "Parameter inference of queueing models for IT systems using end-to-end measurements," Performance Evaluation, vol. 63, no. 1, pp. 3660, 2006.

[25] S. A. Dudin and M. H. Lee, "Analysis of single-server queue with phase-type service and energy harvesting," Mathematical Problems in Engineering, vol. 2016, Article ID 8142743, 16 pages, 2016.

[26] P. Chen, W. H. Zhou, and Y. Zhou, "Equilibrium customer strategies in the queue with threshold policy and setup times," Mathematical Problems in Engineering, vol. 2015, Article ID 361901, 11 pages, 2015. 


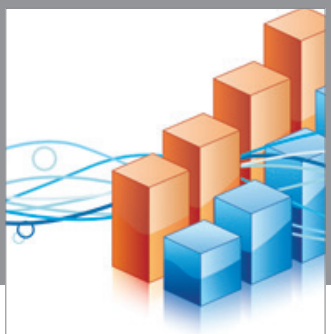

Advances in

Operations Research

vatem alat4

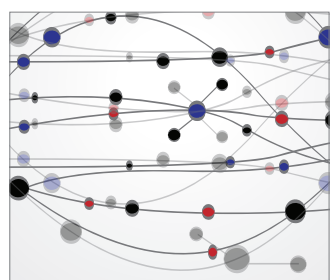

\section{The Scientific} World Journal
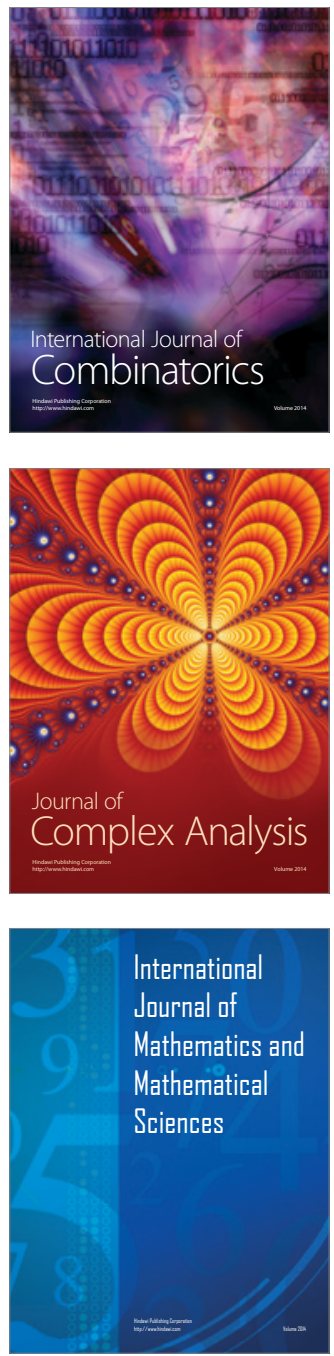
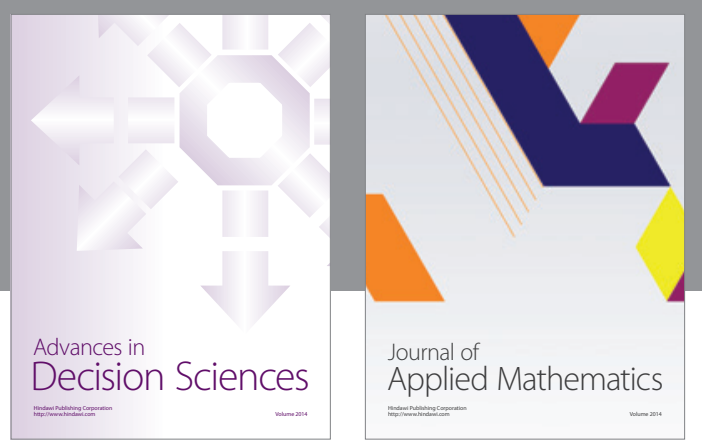

Algebra

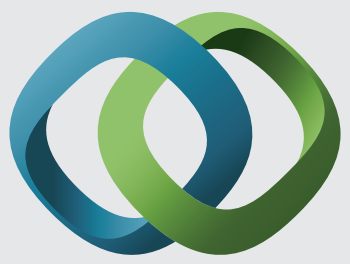

\section{Hindawi}

Submit your manuscripts at

http://www.hindawi.com
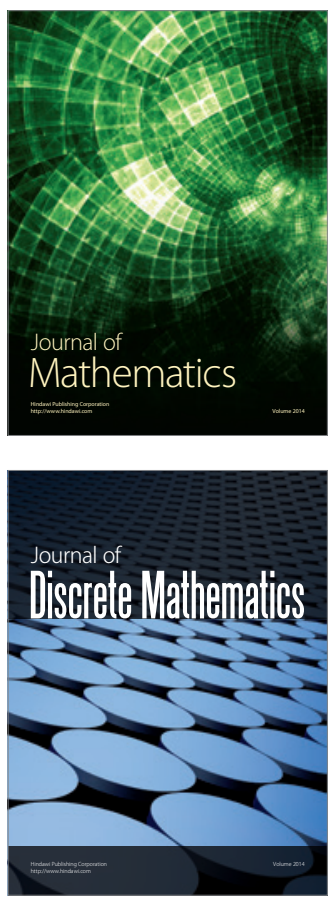

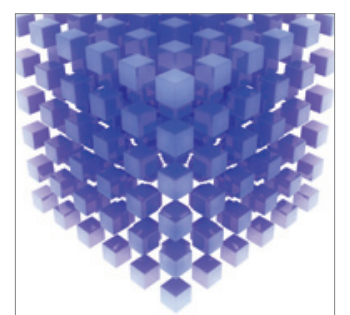

Mathematical Problems in Engineering
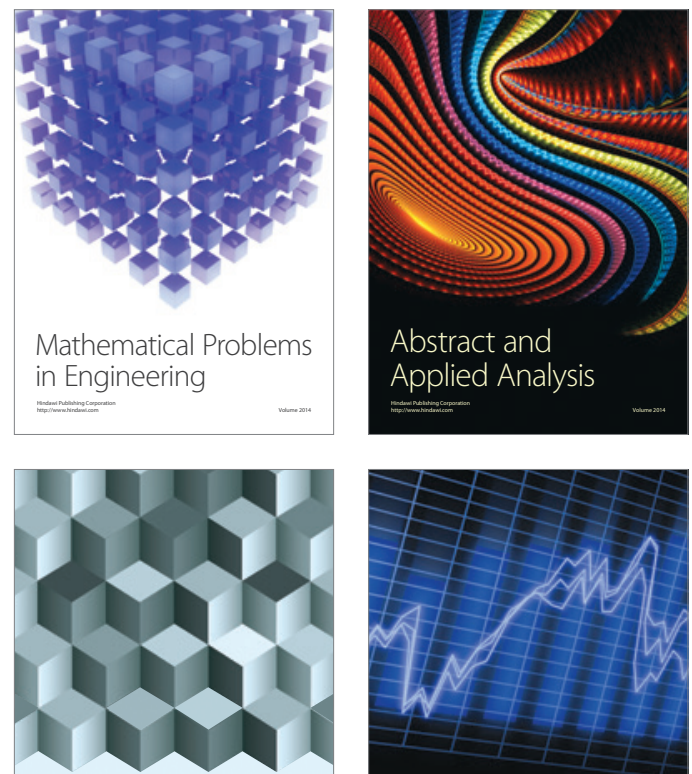

Journal of

Function Spaces

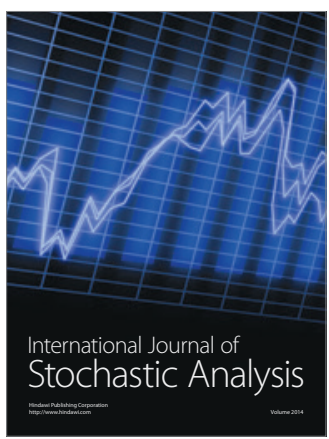

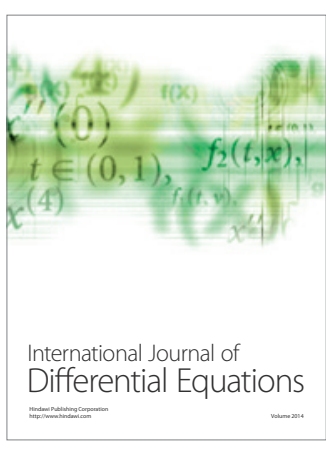
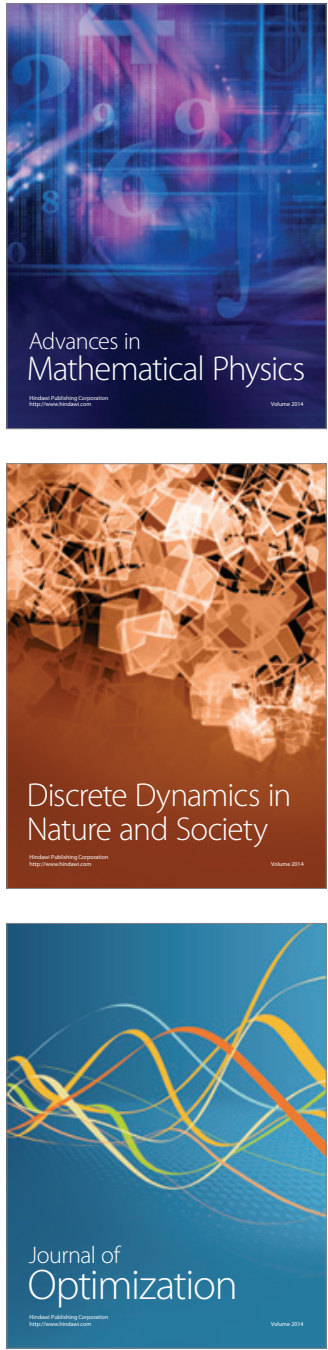